\title{
NEW PRECISION ORBITS OF BRIGHT DOUBLE-LINED SPECTROSCOPIC BINARIES. VIII. HR 1528, HR 6993, 2 SAGITTAE, AND 18 VULPECULAE
}

\author{
Francis C. FeKel $^{1,3}$, Jocelyn Tomkin ${ }^{2}$, and Michael H. Williamson ${ }^{1}$ \\ ${ }^{1}$ Center of Excellence in Information Systems, Tennessee State University, 3500 John A. Merritt Boulevard, \\ Box 9501, Nashville, TN 37209, USA; fekel@evans.tsuniv.edu, michael.h.williamson@ gmail.com \\ 2 Astronomy Department and McDonald Observatory, University of Texas, Austin, TX 78712, USA; jt@ alexis.as.utexas.edu \\ Received 2013 June 28; accepted 2013 August 29; published 2013 October 11
}

\begin{abstract}
Improved orbital elements for four A-star double-lined spectroscopic binaries have been determined with numerous new radial velocities. Three of the four systems, HR 1528, 2 Sge, and 18 Vul, have moderately short orbital periods of 7.05, 7.39, and 9.31 days, respectively, and also have circular or nearly circular orbits. Only HR 6993 with a period of 14.68 days has a significantly eccentric orbit. The close visual companion of 2 Sge has been detected spectroscopically, and its velocity measured. The orbital dimensions $\left(a_{1} \sin i\right.$ and $\left.a_{2} \sin i\right)$ and minimum masses $\left(m_{1} \sin ^{3} i\right.$ and $\left.m_{2} \sin ^{3} i\right)$ of the short-period binary components all have accuracies of $0.5 \%$ or better. We determine basic properties of the individual stars and compare them with solar-abundance evolutionary tracks to estimate their masses. Half of the eight components may be synchronously or pseudosynchronously rotating.
\end{abstract}

Key words: binaries: spectroscopic - stars: fundamental parameters - stars: individual (HR 1528, HR 6993, 2 Sge, 18 Vul)

\section{INTRODUCTION}

With the advances in ground-based optical and near-infrared interferometry over the past decades, there is now a greatly enhanced overlap of the spectroscopic and visual binary domains (Quirrenbach 2001). This has resulted in a significant increase in the number of binary stars that can be resolved with both spectroscopy and astrometry, expanding the possibilities for the direct determination of stellar masses and precise stellar parallaxes from their three-dimensional orbits. There are now more than 30 interferometric visual orbits for systems containing double-lined spectroscopic binaries (Cunha et al. 2007) and at least 23 interferometric binaries that have masses determined to an accuracy of $3 \%$ or better (Torres et al. 2010). The determination of three-dimensional orbits and precise masses (e.g., Hummel et al. 2001; Boden et al. 2006; Fekel et al. 2009a) has resulted in valuable comparisons with stellar evolutionary theory.

In the current edition of the spectroscopic binary orbit catalog, SB9 (Pourbaix et al. 2004), there are still many stars with orbits that have been determined with photographic plate velocities. Such velocities are much less precise than those from modern CCD observations and thus would significantly limit the precision of combined spectroscopic-astrometric orbits. In our series of orbit papers (Tomkin \& Fekel 2006, 2008; Fekel et al. 2009b, 2010, 2011a, 2011b; Fekel \& Williamson 2010), we have acquired new radial velocities from three observatories and used them to redetermine the orbits for 19 bright spectroscopic binaries. Interferometric observations of those systems will lead to three-dimensional orbits and well-determined masses and parallaxes. Some basic information about the four A-star double-lined binaries that are analyzed in this paper is given in Table 1.

\footnotetext{
3 Visiting Astronomer, Kitt Peak National Observatory, National Optical Astronomy Observatory, operated by the Association of Universities for Research in Astronomy, Inc. under cooperative agreement with the National Science Foundation.
}

\section{BRIEF HISTORY}

$$
\text { 2.1. HR } 1528=H D 30453=H I P 22407
$$

Harper (1932a) discovered HR $1528\left[\alpha=04^{\mathrm{h}} 49^{\mathrm{m}} 19^{\mathrm{s}} .08, \delta=\right.$ $32^{\circ} 35^{\prime} 17^{\prime \prime} .5$ (2000)] to be a single-lined spectroscopic binary with a period of 7.0507 days and a near circular orbit. Using Harper's data, Luyten (1936) derived similar elements, while Lucy \& Sweeney (1971) concluded that the orbit was circular. As shown in their Figure 8, Stockton \& Fekel (1992) detected lines of the secondary at red wavelengths. They included Harper's data to improve the period and then computed a circular orbit for the double-lined spectroscopic binary. In 1984, McAlister et al. (1987), using speckle interferometry, announced the discovery of a third component with a separation of $0{ }^{\prime} 04$.

HR 1528 is a well-known metallic-lined star. Appenzeller (1967) gave it an A4m spectral classification, while Bertaud \& Floquet (1967) provided a more detailed description, calling its Ca K line A7 and its metal lines F2. Cowley et al. (1969) classified the $\mathrm{Ca} \mathrm{K}$ line as $\mathrm{A} 8 \mathrm{~m}$. The most complete description of the Am star spectrum was provided by Abt \& Morrell (1995), who assigned types of $\mathrm{A} 7, \mathrm{~F} 0$, and $\mathrm{F} 2$ to the $\mathrm{Ca} \mathrm{K}$, hydrogen, and metal lines, respectively. The star is slowly rotating with $v \sin i$ values of $15 \mathrm{~km} \mathrm{~s}^{-1}$ (Abt \& Moyd 1973; Abt \& Morrell 1995) and $10 \mathrm{~km} \mathrm{~s}^{-1}$ (Stockton \& Fekel 1992).

\subsection{HR $6993=H D 171978=H I P 91322$}

Adams (1915) first reported the radial velocity of HR 6993 $\left[\alpha=18^{\mathrm{h}} 37^{\mathrm{m}} 35^{\mathrm{s}} .96, \delta=-00^{\circ} 18^{\prime} 34^{\prime \prime} .1(2000)\right]$ but did not note any variability. Several decades later Petrie (1948) surveyed stars that were believed to be members of the Ursa Major moving group and immediately discovered its duplicity. Collecting additional high-dispersion observations, he determined an orbit with a period of 14.674 days and an eccentricity of 0.211 for this double-lined early-type spectroscopic binary.

Cowley et al. (1969) and Floquet (1975) classified HR 6993 as A2 V, while Levato \& Abt (1978) concurred in that classification but also noted that the lines were sharp. Geary \& Abt (1970) concluded that the secondary component appears to be an Am 
Table 1

Basic Properties of the Program Stars

\begin{tabular}{lcrcccrr}
\hline \hline Name & HR & HD & Spectral Type & $\begin{array}{c}V^{\mathrm{a}} \\
(\mathrm{mag})\end{array}$ & $\begin{array}{c}B-V^{\mathrm{a}} \\
(\mathrm{mag})\end{array}$ & $\begin{array}{r}\text { Parallax }^{\mathrm{b}} \\
(\mathrm{mas})\end{array}$ & $\begin{array}{r}\text { Period } \\
(\text { days })\end{array}$ \\
\hline$\ldots$ & 1528 & 30453 & Am & 5.84 & 0.250 & 11.94 & 7.05 \\
$\ldots$ & 6993 & 171978 & A2 V & 5.76 & 0.067 & 6.23 & 14.68 \\
2 Sge & 7369 & 182490 & A1 III & 6.27 & 0.075 & 8.92 & 7.39 \\
$18 \mathrm{Vul}$ & 7711 & 191747 & A2 IV & 5.51 & 0.087 & 7.70 & 9.31 \\
\hline
\end{tabular}

Notes.

a Perryman et al. (1997).

b van Leeuwen (2007).

star. Petrie (1948) stated that both components had sharp lines, a result that was quantified by Geary \& Abt (1970), who determined $v \sin i$ values of $<10 \mathrm{~km} \mathrm{~s}^{-1}$ for both components. A larger value of $35 \mathrm{~km} \mathrm{~s}^{-1}$, found by Abt \& Morrell (1995), appears to be from an orbital phase when the lines are partially blended.

\subsection{Sge $=H R 7369=H D 182490=H I P 109647$}

From the measurement of two Mount Wilson Observatory plates, Adams (1912) announced the velocity variability of $2 \mathrm{Sge}$ $\left[\alpha=19^{\mathrm{h}} 24^{\mathrm{m}} 22^{\mathrm{s}} .07, \delta=16^{\circ} 56^{\prime} 15^{\prime \prime} .8(2000)\right]$. Following up on that discovery, at the Dominion Observatory of Ottawa, Young (1917) acquired 44 observations with a one-prism spectrograph, and some of those spectrograms showed lines of the secondary. Using velocities from those plates as well as values from the two discovery observations, Young (1917) determined an initial orbital solution, which he characterized as a first approximation, that resulted in a nearly circular orbit with a period of 7.390 days. Lines of the secondary were so difficult to measure that they were only used to determine the semi-amplitude of the secondary. Young (1917) mentioned that the spectral lines of 2 Sge are fairly sharp and narrow. In his reexamination of 64 orbits, Harper (1935) used six spectra of 2 Sge, obtained at the Dominion Astrophysical Observatory (DAO), to revise the orbital period to 7.3919 days. The binary was one of 77 systems with small orbital eccentricities that Luyten (1936) reanalyzed. Using the 44 Dominion Observatory observations only, he adopted a circular orbit and recomputed the elements.

Analysis of the Hipparcos data resulted in the discovery of a faint, close visual companion to 2 Sge (Perryman et al. 1997). Fabricius \& Makarov (2000) determined a Tycho $V$-band difference of 3.8 mag, while Roberts (2011) measured a Bessel I-band difference of $3.4 \mathrm{mag}$. Mason et al. (1999) followed up the discovery observation with a very weak ground-based detection in 1996. More recently, Roberts (2011) resolved the components again, showing that the pair were still separated by $0^{\prime \prime} .4$ but had rotated $11^{\circ}$ in $11 \mathrm{yr}$.

Over the years, a number of different spectral types have been assigned to 2 Sge. Osawa (1959) classified 2 Sge as A2 III? and noted that its metal lines had an A3 class. Cowley et al. (1969) called the Ca K line A1 and stated that the star's metal lines were marginally enhanced, indicating that 2 Sge is a mild metallic-lined A star. Bidelman (Abt \& Bidelman 1969) concluded that it is a probable Am star, while Bertaud (1970) classified it as a full-fledged Am star with its Ca K line being A2 and its metal lines A7. Other classifiers have found no spectrum peculiarities. Levato (1975) assigned a spectral type of A2 III-IV, while Abt \& Morrell (1995) classified its spectral type as A1 III. In addition, Cucchiaro et al. (1978) obtained an ultraviolet spectrum of 2 Sge with the sky survey telescope of the TD1 satellite and classified the star as an A2 giant. Stickland (1973) performed a spectroscopic abundance analysis of both components and concluded that Fe was overabundant in both stars. Using four Fe I line pairs in the blue region of the spectrum, Petrie (1950) estimated a magnitude difference of 0.65 for the short-period binary. Levato (1975) determined a $v \sin i$ value of $50 \mathrm{~km} \mathrm{~s}^{-1}$, while Abt \& Morrell (1995) found a smaller value of $35 \mathrm{~km} \mathrm{~s}^{-1}$.

\section{4. $18 \mathrm{Vul}=H R 7711=H D 191747=H I P 99404$}

Velocity measurements of three spectroscopic plates, obtained at Mount Wilson Observatory, led Adams (1915) to announce the binary nature of 18 Vul $\left[\alpha=20^{\mathrm{h}} 10^{\mathrm{m}} 33.54, \delta=\right.$ $\left.26^{\circ} 54^{\prime} 15^{\prime \prime} .0(2000)\right]$ with the components having a velocity separation of $150 \mathrm{~km} \mathrm{~s}^{-1}$ on one plate. Following up on this discovery, Harper (1918) collected 62 usable spectra at the Dominion Observatory at Ottawa from which he computed a nearly circular orbit with a period of 9.316 days. With the acquisition of five additional velocities, he later decreased the period to 9.314 days (Harper 1935). Luyten (1936) reanalyzed the velocities and considered the orbit to be circular.

Osawa (1959) gave a spectral type of A3 III for the combined system. That result was followed by Svolopoulos (1961), who classified a number of stars in open clusters and included $18 \mathrm{Vul}$ as A1 IV. Additional spectral types of A3 V (Cowley et al. 1969) and A2 IV (Abt \& Morrell 1995) have been given. Cucchiaro et al. (1978) examined an ultraviolet spectrum of 18 Vul with the sky survey telescope of the TDl satellite and classified the star as an A3 dwarf from ultraviolet criteria. Bidelman (Abt \& Bidelman 1969) concluded that its spectrum was that of a normal A star with no peculiarities.

Breger (1969) included $18 \mathrm{Vul}$ in a search for $\delta$ Scuti variables among more than 200 bright field stars. His limited observations indicated that the star was constant at a level of $2 \mathrm{mmag}$. However, later photometric observations by Belmonte \& Roca Cortés (1989) revealed low-amplitude variability of $4 \mathrm{mmag}$ with a period of $2.9 \mathrm{hr}$. Although classified as a $\delta$ Scuti variable, its position is somewhat blueward of the blue edge of the $\delta$ Scuti instability strip (Rodríguez et al. 1994).

\section{OBSERVATIONS AND RADIAL VELOCITIES}

We acquired spectroscopic observations of the four program stars at three different observatories. From 2002 through 2007, we collected spectra at McDonald Observatory with the $2.1 \mathrm{~m}$ telescope, the Sandiford Cassegrain echelle spectrograph (McCarthy et al. 1993), and a Reticon CCD. The wavelength range covered by those spectrograms is 5700-7000 $\AA$ and the resolving power is 49,000 .

From 2004 through 2012, we also obtained spectrograms of the binaries at Kitt Peak National Observatory (KPNO) with the coudé feed telescope and coudé spectrograph. The vast majority of the observations were made with a Texas Instruments (TI) CCD detector. Those spectra are mostly centered at $6430 \AA$, cover a wavelength range of $84 \AA$, and have a resolution of $0.21 \AA$ or a resolving power of just over 30,000 . A few spectra were centered in the blue at $4500 \AA$ but with the same wavelength range of $84 \AA$ and resolution, producing a resolving power of 21,000 . When the TI detector was unavailable in 2008 September, we used instead a Tektronics CCD, designated T1KA. With that CCD the spectrum was centered at $6400 \AA$, the wavelength coverage increased to $172 \AA$, and the resolving power decreased to 19,000 . The TI CCD was retired from 
service in 2010 September. As a result, our observations from that point forward were obtained with a new CCD, made by Semiconductor Technology Associates and given the designation STA2. It consists of a $2600 \times 4000$ array of $12 \mu \mathrm{m}$ pixels. With STA2 the spectrum once again was centered at $6430 \AA$, and the size of the detector produced a wavelength range of $336 \AA$. The spectrograph slit was set so that the STA2 spectra have the same resolution as those acquired with the TI CCD although there is some worsening of the resolution at both ends of the STA2 spectra. Additional information about our McDonald and KPNO observations is given in Tomkin \& Fekel (2006).

Finally, from 2003 through 2013, we collected an extensive number of spectra with the Tennessee State University $2 \mathrm{~m}$ Automatic Spectroscopic Telescope (AST) and a fiber-fed echelle spectrograph, situated at Fairborn Observatory near Washington Camp in the Patagonia Mountains of southeastern Arizona (Eaton \& Williamson 2004, 2007). Through 2011 June the detector was a $2048 \times 4096$ SITe ST-002A CCD with $15 \mu \mathrm{m}$ pixels. Eaton \& Williamson (2007) have discussed reduction of the raw spectra and their wavelength calibration. Those AST echelle spectrograms have 21 orders that cover the wavelength range 4920-7100 $\AA$ with an average resolution of $0.17 \AA$. The typical signal-to-noise ratio of these observations is $\sim 80$ at $6000 \AA$.

In the summer of 2011 the SITe CCD detector and dewar were replaced with a Fairchild 486 CCD with a $4000 \times 4000$ array of $15 \mu \mathrm{m}$ pixels, which required a new readout electronics package, and a new dewar (Fekel et al. 2013). The echelle spectrograms that were obtained with this new detector have 48 orders, covering the wavelength range $3800-8260 \AA$. Because different diameter fibers were used at various times, the resolution of the new echelle spectra is either 0.24 or $0.4 \AA$, which resulted in signal-to-noise ratios that ranged from 80 to 150 at $6000 \AA$.

Tomkin \& Fekel (2006) have discussed in detail the procedures that we have used to measure the McDonald and KPNO radial velocities. While the McDonald velocities are on an absolute scale, the KPNO velocities are relative velocities that have been determined by cross-correlation with respect to stars with constant radial velocities. For the KPNO red wavelength spectra, we used the IAU standards 10 Tau, HR 5694, and HR 7560 with velocities adopted from Scarfe et al. (1990). For the blue wavelength spectra, we employed several early-A stars, 68 Tau, HR 7773, and HR 8404, from a list of such stars that have been observed to identify constant velocity early-type stars (Fekel 1999). From our unpublished observations, our adopted velocities for those three stars are $39.0,-1.1$, and $0.1 \mathrm{~km} \mathrm{~s}^{-1}$, respectively.

Fekel et al. (2009b) provided an extensive general description of velocity measurement of the Fairborn AST spectra. The fact that HR 1528 is an Am star enabled us to use our line list for solar-type stars, which includes mostly neutral Fe lines, while for HR 6993 and 18 Vul we chose our line list for early- and midA type stars, which consists mostly of singly ionized lines of $\mathrm{Fe}$, $\mathrm{Si}$, and $\mathrm{Ti}$. The lines of the $2 \mathrm{Sge}$ short-period binary are narrow, enabling both components to be measured with either the solar or A-star line lists. Because we spectroscopically detected lines of the much fainter Hipparcos close visual companion, which could only be measured with the solar line list, we chose to measure all three components of 2 Sge with that list. A Gaussian function, which is quite adequate to fit the features of narrowlined stars, was used to determine the velocities of HR 1528 and
HR 6993. However, because of the significantly larger rotational broadening of the $18 \mathrm{Vul}$ components and the blending problems that sometimes occurred in the 2 Sge system because of lines of the third component, we used a rotational broadening function (Lacy \& Fekel 2011) to fit the lines of those systems.

Like the velocities from McDonald, the resulting Fairborn velocities are absolute rather than relative velocities. Our unpublished measurements of several IAU standard solar-type stars indicate that the Fairborn Observatory velocities taken with the SITe CCD have a small zero-point offset of $-0.3 \mathrm{~km} \mathrm{~s}^{-1}$ relative to the velocities of Scarfe et al. (1990). Starting in the fall of 2011, velocities from spectra obtained with the new CCD system have a zero-point offset $-0.6 \mathrm{~km} \mathrm{~s}^{-1}$ relative to those of Scarfe et al. (1990). Thus, we added either 0.3 or $0.6 \mathrm{~km} \mathrm{~s}^{-1}$, depending on which detector was used, to each measured velocity.

Fekel et al. (2011a) reaffirmed that despite the different methods of reduction, the KPNO velocities are on the same scale as the absolute velocities of McDonald to within a precision of $0.1-0.2 \mathrm{~km} \mathrm{~s}^{-1}$, as was found by Tomkin \& Fekel (2006). Thus, with the Fairborn zero-point corrections, the zero-point velocities of all three observatories should be essentially the same. Even if there was a more significant offset between the various data sets, it would simply slightly increase the uncertainty of the orbital elements since the number of Fairborn velocities of each system is at least three times larger than those from KPNO or McDonald.

\section{DETERMINATION OF SPECTROSCOPIC ORBITS AND RESULTS}

To determine the orbital elements we have used several computer programs. The program BISP (Wolfe et al. 1967), which implements a slightly modified version of the Wilsing-Russell method, has been used to determine initial orbits. With the differential corrections program SB1 (Barker et al. 1967), we then determined eccentric orbits. For a simultaneous solution of the two components, we used SB2, which is a slightly modified version of SB1. Finally, when appropriate, we computed circular orbits with the differential corrections programs SB1C and SB2C (D. Barlow 1998, private communication).

As mentioned earlier, our new spectra have been acquired at three observatories. The various detectors that we have used have different wavelength ranges, and so different numbers of lines have been measured to determine an average radial velocity. Also, the binary components usually have unequal line strengths and/or widths. These differences produce unequal velocity precisions. Therefore, when possible, we computed individual orbital solutions for the data sets of each component from each observatory. The variances of those individual solutions are inversely proportional to the weights that we assigned to the velocities in those different sets.

\section{1. $H R 1528$}

At Fairborn Observatory between 2003 and 2013, we acquired 95 spectrograms that show double lines. An additional 10 observations were obtained from 2004 to 2007 at McDonald Observatory and from 2006 through 2008 five more at KPNO were also collected. All of our new velocities are listed in Table 2.

To confirm the conclusion of Luyten (1936) and Stockton \& Fekel (1992) that the orbit is circular, we analyzed the numerous Fairborn velocities of the much stronger primary lines. The resulting eccentricity of $0.00051 \pm 0.00033$ is very small and 
Table 2

Radial Velocities of HR 1528

\begin{tabular}{|c|c|c|c|c|c|c|c|c|}
\hline $\begin{array}{l}\text { Hel. Julian Date } \\
\text { (HJD }-2,400,000)\end{array}$ & Phase & $\begin{array}{c}V_{1} \\
\left(\mathrm{~km} \mathrm{~s}^{-1}\right)\end{array}$ & $\begin{array}{l}(O-C)_{1} \\
\left(\mathrm{~km} \mathrm{~s}^{-1}\right)\end{array}$ & $\mathrm{Wt}_{1}$ & $\begin{array}{c}V_{2} \\
\left(\mathrm{~km} \mathrm{~s}^{-1}\right)\end{array}$ & $\begin{array}{l}(O-C)_{2} \\
\left(\mathrm{~km} \mathrm{~s}^{-1}\right)\end{array}$ & $\mathrm{Wt}_{2}$ & Source $^{\mathrm{a}}$ \\
\hline 52966.931 & 0.931 & 70.6 & -0.1 & 1.0 & -64.9 & 0.5 & 0.07 & Fair \\
\hline 52967.958 & 0.077 & 69.4 & 0.1 & 1.0 & -63.6 & -0.4 & 0.07 & Fair \\
\hline 53031.806 & 0.132 & 56.7 & 0.0 & 1.0 & -44.3 & -0.2 & 0.07 & Fair \\
\hline 53057.810 & 0.821 & 42.1 & 0.0 & 1.0 & -22.2 & -0.1 & 0.07 & Fair \\
\hline 53108.605 & 0.025 & 75.4 & 0.0 & 0.8 & -73.0 & -0.4 & 0.02 & $\mathrm{McD}$ \\
\hline 53109.635 & 0.171 & 45.1 & 0.0 & 0.8 & -27.0 & -0.5 & 0.02 & $\mathrm{McD}$ \\
\hline 53277.810 & 0.022 & 75.5 & 0.0 & 1.0 & -72.6 & 0.1 & 0.07 & Fair \\
\hline 53287.031 & 0.330 & -12.0 & 0.0 & 1.0 & 59.9 & -0.3 & 0.07 & Fair \\
\hline 53293.008 & 0.178 & 42.6 & -0.1 & 1.0 & -22.1 & 0.8 & 0.07 & Fair \\
\hline 53301.994 & 0.452 & -40.0 & 0.1 & 1.0 & 103.6 & 0.7 & 0.07 & Fair \\
\hline 53311.915 & 0.859 & 54.2 & -0.2 & 1.0 & -41.1 & -0.5 & 0.07 & Fair \\
\hline 53313.007 & 0.014 & 75.9 & 0.0 & 1.0 & -74.0 & -0.7 & 0.07 & Fair \\
\hline 53319.927 & 0.996 & 76.1 & 0.0 & 1.0 & -73.9 & -0.3 & 0.07 & Fair \\
\hline 53334.059 & 1.000 & 75.9 & -0.2 & 1.0 & -74.3 & -0.7 & 0.07 & Fair \\
\hline 53340.886 & 0.968 & 75.0 & 0.1 & 1.0 & -71.5 & 0.3 & 0.07 & Fair \\
\hline 53351.930 & 0.535 & -41.3 & 0.1 & 1.0 & 105.3 & 0.5 & 0.07 & Fair \\
\hline 53358.786 & 0.507 & -42.9 & -0.2 & 1.0 & 106.5 & -0.3 & 0.07 & Fair \\
\hline 53385.889 & 0.351 & -18.7 & -0.2 & 1.0 & 69.3 & -0.8 & 0.07 & Fair \\
\hline 53395.880 & 0.768 & 23.5 & 0.2 & 1.0 & 6.2 & -0.4 & 0.07 & Fair \\
\hline 53400.738 & 0.457 & -40.5 & 0.1 & 0.8 & 103.1 & -0.5 & 0.02 & $\mathrm{McD}$ \\
\hline 53401.757 & 0.601 & -31.1 & 0.0 & 0.8 & 89.1 & -0.1 & 0.02 & $\mathrm{McD}$ \\
\hline 53489.598 & 0.060 & 72.0 & 0.0 & 0.8 & -67.6 & -0.2 & 0.02 & $\mathrm{McD}$ \\
\hline 53662.946 & 0.645 & -20.0 & -0.2 & 0.8 & 70.4 & -1.7 & 0.02 & $\mathrm{McD}$ \\
\hline 53663.852 & 0.773 & 25.6 & 0.3 & 0.8 & 6.0 & 2.5 & 0.02 & $\mathrm{McD}$ \\
\hline 53774.661 & 0.489 & -42.6 & 0.0 & 0.8 & 106.3 & -0.4 & 0.02 & $\mathrm{McD}$ \\
\hline 53775.668 & 0.632 & -23.5 & 0.0 & 0.8 & 77.4 & -0.3 & 0.02 & $\mathrm{McD}$ \\
\hline 54002.968 & 0.869 & 56.9 & -0.2 & 0.8 & -44.9 & -0.2 & 0.02 & KPNO \\
\hline 54003.928 & 0.005 & 76.1 & 0.0 & 0.8 & -73.1 & 0.5 & 0.02 & KPNO \\
\hline 54106.717 & 0.583 & -34.8 & 0.0 & 0.8 & 94.4 & -0.4 & 0.02 & $\mathrm{McD}$ \\
\hline 54364.920 & 0.203 & 34.0 & 0.2 & 0.8 & -8.9 & 0.5 & 0.02 & KPNO \\
\hline 54369.956 & 0.918 & 67.9 & -0.4 & 0.8 & -61.3 & 0.5 & 0.02 & KPNO \\
\hline 54526.659 & 0.142 & 53.9 & 0.0 & 0.8 & -40.8 & -0.9 & 0.02 & KPNO \\
\hline 55241.774 & 0.564 & -38.3 & -0.3 & 1.0 & 99.4 & -0.2 & 0.07 & Fair \\
\hline 55276.730 & 0.522 & -42.0 & 0.2 & 1.0 & 105.4 & -0.7 & 0.07 & Fair \\
\hline 55290.685 & 0.501 & -42.8 & 0.0 & 1.0 & 107.0 & 0.1 & 0.07 & Fair \\
\hline 55302.619 & 0.194 & 37.1 & -0.1 & 1.0 & -15.3 & -0.8 & 0.07 & Fair \\
\hline 55478.744 & 0.173 & 44.2 & -0.1 & 1.0 & -24.8 & 0.5 & 0.07 & Fair \\
\hline 55485.981 & 0.200 & 35.1 & -0.1 & 1.0 & -12.2 & -0.7 & 0.07 & Fair \\
\hline 55498.011 & 0.906 & 65.9 & -0.1 & 1.0 & -59.2 & -1.0 & 0.07 & Fair \\
\hline 55513.935 & 0.164 & 47.2 & 0.0 & 1.0 & -29.6 & 0.1 & 0.07 & Fair \\
\hline 55521.925 & 0.297 & -0.8 & 0.0 & 1.0 & 43.0 & -0.1 & 0.07 & Fair \\
\hline 55544.984 & 0.568 & -37.8 & -0.3 & 1.0 & 99.5 & 0.6 & 0.07 & Fair \\
\hline 55565.889 & 0.533 & -41.5 & 0.0 & 1.0 & 105.1 & 0.1 & 0.07 & Fair \\
\hline 55573.910 & 0.670 & -12.0 & -0.1 & 1.0 & 59.3 & -0.8 & 0.07 & Fair \\
\hline 55579.801 & 0.506 & -42.7 & 0.0 & 1.0 & 106.9 & 0.0 & 0.07 & Fair \\
\hline 55586.821 & 0.501 & -42.8 & 0.0 & 1.0 & 107.7 & 0.8 & 0.07 & Fair \\
\hline 55600.833 & 0.489 & -42.5 & 0.1 & 1.0 & 106.3 & -0.4 & 0.07 & Fair \\
\hline 55618.744 & 0.029 & 75.0 & -0.1 & 1.0 & -73.1 & -0.9 & 0.07 & Fair \\
\hline 55626.772 & 0.167 & 46.2 & 0.1 & 1.0 & -28.8 & -0.7 & 0.07 & Fair \\
\hline 55635.647 & 0.426 & -36.6 & -0.1 & 1.0 & 96.7 & -0.7 & 0.07 & Fair \\
\hline 55645.676 & 0.848 & 51.2 & 0.1 & 1.0 & -35.3 & 0.4 & 0.07 & Fair \\
\hline 55651.691 & 0.702 & -1.2 & 0.0 & 1.0 & 42.8 & -0.9 & 0.07 & Fair \\
\hline 55842.826 & 0.810 & 38.4 & 0.0 & 1.0 & -17.0 & -0.6 & 0.07 & Fair \\
\hline 55848.868 & 0.666 & -13.2 & -0.1 & 1.0 & 61.6 & -0.3 & 0.07 & Fair \\
\hline 55854.768 & 0.503 & -42.9 & -0.1 & 1.0 & 106.9 & 0.0 & 0.07 & Fair \\
\hline 55862.830 & 0.647 & -19.4 & -0.1 & 1.0 & 70.8 & -0.4 & 0.07 & Fair \\
\hline 55882.781 & 0.476 & -41.9 & 0.2 & 1.0 & 106.4 & 0.5 & 0.07 & Fair \\
\hline 55893.867 & 0.049 & 73.2 & -0.2 & 1.0 & -69.4 & 0.1 & 0.07 & Fair \\
\hline 55903.876 & 0.468 & -41.4 & 0.2 & 1.0 & 105.5 & 0.4 & 0.07 & Fair \\
\hline 55921.670 & 0.992 & 76.0 & 0.0 & 1.0 & -73.1 & 0.4 & 0.07 & Fair \\
\hline 55929.872 & 0.155 & 49.9 & -0.2 & 1.0 & -34.0 & 0.1 & 0.07 & Fair \\
\hline 55932.933 & 0.589 & -33.7 & 0.0 & 1.0 & 92.6 & -0.5 & 0.07 & Fair \\
\hline 55936.918 & 0.154 & 50.3 & 0.0 & 1.0 & -34.1 & 0.3 & 0.07 & Fair \\
\hline 55946.801 & 0.556 & -39.2 & -0.1 & 1.0 & 102.0 & 0.6 & 0.07 & Fair \\
\hline
\end{tabular}


Table 2

(Continued)

\begin{tabular}{|c|c|c|c|c|c|c|c|c|}
\hline $\begin{array}{l}\text { Hel. Julian Date } \\
\text { (HJD - 2,400,000) }\end{array}$ & Phase & $\begin{array}{c}V_{1} \\
\left(\mathrm{~km} \mathrm{~s}^{-1}\right)\end{array}$ & $\begin{array}{c}(O-C)_{1} \\
\left(\mathrm{~km} \mathrm{~s}^{-1}\right)\end{array}$ & $\mathrm{Wt}_{1}$ & $\begin{array}{c}V_{2} \\
\left(\mathrm{~km} \mathrm{~s}^{-1}\right)\end{array}$ & $\begin{array}{c}(O-C)_{2} \\
\left(\mathrm{~km} \mathrm{~s}^{-1}\right)\end{array}$ & $\mathrm{Wt}_{2}$ & Source $^{\mathrm{a}}$ \\
\hline 55955.802 & 0.833 & 46.2 & 0.1 & 1.0 & -28.1 & 0.0 & 0.07 & Fair \\
\hline 55962.858 & 0.833 & 46.2 & -0.2 & 1.0 & -28.6 & -0.1 & 0.07 & Fair \\
\hline 55970.830 & 0.964 & 74.6 & 0.0 & 1.0 & -71.2 & 0.1 & 0.07 & Fair \\
\hline 55977.701 & 0.938 & 71.8 & 0.1 & 1.0 & -66.2 & 0.8 & 0.07 & Fair \\
\hline 55984.690 & 0.930 & 70.5 & 0.1 & 1.0 & -64.6 & 0.4 & 0.07 & Fair \\
\hline 55996.756 & 0.641 & -21.3 & -0.3 & 1.0 & 74.3 & 0.5 & 0.07 & Fair \\
\hline 56016.675 & 0.466 & -41.5 & -0.1 & 1.0 & 105.2 & 0.3 & 0.07 & Fair \\
\hline 56027.648 & 0.022 & 75.5 & 0.0 & 1.0 & -73.0 & -0.3 & 0.07 & Fair \\
\hline 56029.657 & 0.307 & -4.5 & -0.3 & 1.0 & 48.9 & 0.5 & 0.07 & Fair \\
\hline 56193.789 & 0.585 & -34.5 & -0.1 & 1.0 & 94.3 & 0.1 & 0.07 & Fair \\
\hline 56200.849 & 0.587 & -34.2 & 0.0 & 1.0 & 93.7 & -0.2 & 0.07 & Fair \\
\hline 56203.917 & 0.022 & 75.5 & 0.0 & 1.0 & -72.6 & 0.2 & 0.07 & Fair \\
\hline 56210.890 & 0.011 & 75.8 & -0.2 & 1.0 & -73.6 & -0.2 & 0.07 & Fair \\
\hline 56215.737 & 0.698 & -2.1 & 0.2 & 1.0 & 45.8 & 0.3 & 0.07 & Fair \\
\hline 56217.876 & 0.002 & 76.1 & 0.0 & 1.0 & -74.0 & -0.4 & 0.07 & Fair \\
\hline 56223.872 & 0.852 & 52.1 & -0.1 & 1.0 & -37.5 & -0.2 & 0.07 & Fair \\
\hline 56225.697 & 0.111 & 62.2 & -0.1 & 1.0 & -53.6 & -1.0 & 0.07 & Fair \\
\hline 56226.858 & 0.275 & 7.3 & 0.1 & 1.0 & 32.0 & 0.9 & 0.07 & Fair \\
\hline 56229.838 & 0.698 & -2.3 & 0.1 & 1.0 & 46.0 & 0.4 & 0.07 & Fair \\
\hline 56231.681 & 0.960 & 74.3 & 0.1 & 1.0 & -70.3 & 0.4 & 0.07 & Fair \\
\hline 56232.694 & 0.103 & 64.0 & 0.0 & 1.0 & -55.8 & -0.5 & 0.07 & Fair \\
\hline 56236.835 & 0.690 & -5.0 & 0.1 & 1.0 & 49.7 & 0.1 & 0.07 & Fair \\
\hline 56237.825 & 0.831 & 45.7 & 0.1 & 1.0 & -27.0 & 0.3 & 0.07 & Fair \\
\hline 56243.036 & 0.570 & -37.0 & 0.1 & 1.0 & 98.9 & 0.5 & 0.07 & Fair \\
\hline 56250.642 & 0.649 & -18.7 & 0.0 & 1.0 & 70.3 & 0.0 & 0.07 & Fair \\
\hline 56255.888 & 0.393 & -29.7 & 0.1 & 1.0 & 86.8 & -0.4 & 0.07 & Fair \\
\hline 56260.761 & 0.084 & 68.0 & 0.0 & 1.0 & -61.6 & -0.2 & 0.07 & Fair \\
\hline 56264.916 & 0.673 & -11.0 & 0.0 & 1.0 & 58.6 & 0.0 & 0.07 & Fair \\
\hline 56266.864 & 0.949 & 73.1 & 0.0 & 1.0 & -68.0 & 1.1 & 0.07 & Fair \\
\hline 56267.848 & 0.089 & 67.1 & 0.1 & 1.0 & -60.5 & -0.6 & 0.07 & Fair \\
\hline 56272.838 & 0.797 & 33.9 & 0.1 & 1.0 & -9.5 & -0.1 & 0.07 & Fair \\
\hline 56273.830 & 0.937 & 71.8 & 0.2 & 1.0 & -66.1 & 0.6 & 0.07 & Fair \\
\hline 56274.850 & 0.082 & 68.5 & 0.1 & 1.0 & -62.3 & -0.4 & 0.07 & Fair \\
\hline 56281.737 & 0.059 & 72.2 & 0.1 & 1.0 & -66.9 & 0.6 & 0.07 & Fair \\
\hline 56288.882 & 0.072 & 70.3 & 0.2 & 1.0 & -64.1 & 0.4 & 0.07 & Fair \\
\hline 56295.930 & 0.072 & 70.4 & 0.2 & 1.0 & -64.1 & 0.5 & 0.07 & Fair \\
\hline 56302.929 & 0.064 & 71.6 & 0.3 & 1.0 & -65.7 & 0.6 & 0.07 & Fair \\
\hline 56310.889 & 0.193 & 37.4 & 0.0 & 1.0 & -15.0 & -0.2 & 0.07 & Fair \\
\hline 56311.874 & 0.333 & -12.9 & 0.1 & 1.0 & 61.2 & -0.4 & 0.07 & Fair \\
\hline 56324.849 & 0.173 & 44.0 & -0.2 & 1.0 & -24.9 & 0.3 & 0.07 & Fair \\
\hline 56331.825 & 0.163 & 47.7 & 0.0 & 1.0 & -29.7 & 0.8 & 0.07 & Fair \\
\hline 56340.815 & 0.438 & -38.0 & 0.3 & 1.0 & 100.6 & 0.5 & 0.07 & Fair \\
\hline 56348.802 & 0.570 & -37.1 & 0.0 & 1.0 & 97.8 & -0.4 & 0.07 & Fair \\
\hline 56349.798 & 0.712 & 2.5 & 0.0 & 1.0 & 38.8 & 0.6 & 0.07 & Fair \\
\hline 56358.760 & 0.983 & 75.6 & -0.1 & 1.0 & -73.3 & -0.2 & 0.07 & Fair \\
\hline 56384.686 & 0.660 & -15.4 & -0.1 & 1.0 & 65.1 & -0.1 & 0.07 & Fair \\
\hline
\end{tabular}

Note. ${ }^{\text {a }}$ Fair $=$ Fairborn Observatory, $\mathrm{McD}=\mathrm{McDonald}$ Observatory, $\mathrm{KPNO}=$ Kitt Peak National Observatory.

consistent with the assumption of a circular orbit (Lucy \& Sweeney 1971). Because of the small number of velocities from McDonald and especially KPNO, we combined the velocities from those two observatories and obtained separate circular orbit solutions for the primary and secondary. Two more independent circular orbit solutions were computed with the Fairborn velocities, one for each component. From those results we determined weights of $1.0,0.8$, and 0.8 for the Fairborn, McDonald, and KPNO velocities of the primary, respectively, and $0.07,0.02$, and 0.02 for the secondary velocities from the same three observatories. The four individual solutions have center-of-mass velocities within $0.2 \mathrm{~km} \mathrm{~s}^{-1}$ of each other. Thus, we computed a simultaneous circular orbit solution of the weighted primary and secondary velocities from the three observatories, which produced a period of 7.050869 days.

In principle, earlier radial velocities of HR 1528 could be used to improve the precision of the period and possibly the other elements. Thus, we examined the KPNO primary velocities of Stockton \& Fekel (1992). An orbital solution of those data alone produces a center-of-mass velocity that differs by $1.2 \mathrm{~km} \mathrm{~s}^{-1}$ from the solution of our new data, and results in weights of 0.03 for the observations. Combining those velocities, shifted by $-1.2 \mathrm{~km} \mathrm{~s}^{-1}$, with our new velocities from Fairborn, McDonald, and KPNO, we obtained a circular solution with orbital elements that do not differ significantly from the elements computed with just our new velocities. In particular, the period, whose precision 
Table 3

Orbital Elements and Related Parameters of HR 1528

\begin{tabular}{lcc}
\hline \hline Parameter & Stockton \& Fekel (1992) & This Study \\
\hline$P($ days $)$ & $7.050933 \pm 0.000024$ & $7.0508687 \pm 0.0000023$ \\
$T_{0}(\mathrm{HJD})$ & 2448158.713 & $2454673.72440 \pm 0.00043$ \\
$e$ & 0.0 (adopted) & 0.0 (adopted) \\
$K_{1}\left(\mathrm{~km} \mathrm{~s}^{-1}\right)$ & $58.9 \pm 0.3$ & $59.442 \pm 0.017$ \\
$K_{2}\left(\mathrm{~km} \mathrm{~s}^{-1}\right)$ & $89.7 \pm 0.7$ & $90.283 \pm 0.065$ \\
$\gamma\left(\mathrm{km} \mathrm{s}^{-1}\right)$ & $17.8 \pm 0.1$ & $16.654 \pm 0.012$ \\
$m_{1} \sin ^{3} i\left(M_{\odot}\right)$ & $1.45 \pm 0.02$ & $1.4786 \pm 0.0024$ \\
$m_{2} \sin ^{3} i\left(M_{\odot}\right)$ & $0.95 \pm 0.01$ & $0.9735 \pm 0.0010$ \\
$a_{1} \sin i\left(10^{6} \mathrm{~km}\right)$ & $5.71 \pm 0.03$ & $5.7633 \pm 0.0016$ \\
$a_{2} \sin i\left(10^{6} \mathrm{~km}\right)$ & $8.70 \pm 0.07$ & $8.7536 \pm 0.0063$ \\
Standard error of an unit weight observation $\left(\mathrm{km} \mathrm{s}^{-1}\right)$ & $\ldots$ & 0.13 \\
\hline
\end{tabular}

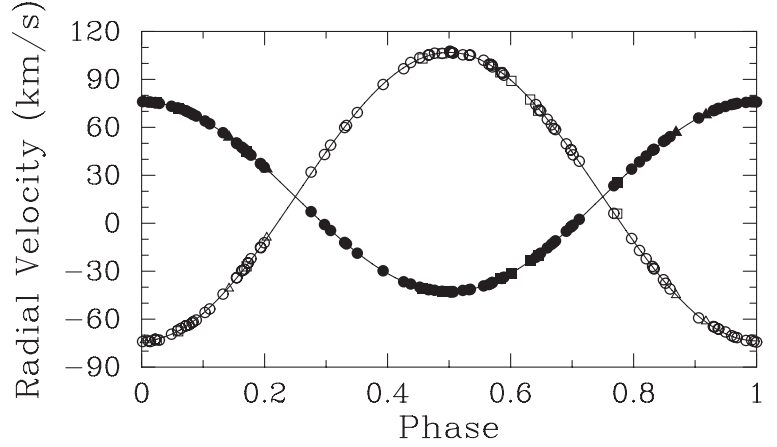

Figure 1. Radial velocities of HR 1528 compared with the computed velocity curves. Filled and open symbols represent the primary and secondary, respectively. Circles $=$ Fairborn Observatory, triangles $=\mathrm{KPNO}$, and squares $=\mathrm{Mc}$ Donald Observatory. Zero phase is a time of maximum velocity of the primary.

could benefit from the longer time span of the four data sets, is changed by less than $1 \sigma$. Thus, we have chosen to adopt the simultaneous circular orbit solution, determined with only our new primary and secondary velocities. Table 3 lists those elements. For a circular orbit the element $T$, a time of periastron passage, is undefined. Thus, as recommended by Batten et al. (1989), $T_{0}$, a time of maximum velocity for the primary, is used instead. Figure 1 compares our primary and secondary velocities from our three observatories with the calculated velocity curves. Figure 2 plots the velocity residuals versus phase for the primary and secondary. As expected from the large magnitude difference between the two components (Section 5.1), the typical residual of the secondary is much greater than that of the primary.

In addition to our new elements, Table 3 lists the elements of Stockton \& Fekel (1992). A comparison shows that the values of the elements are in good agreement, but our newly derived elements are five to 10 times more precise. The systemic velocities of the two solutions in Table 3 differ by $1.2 \mathrm{~km} \mathrm{~s}^{-1}$, $-17.8 \pm 0.1 \mathrm{~km} \mathrm{~s}^{-1}$ versus $-16.65 \pm 0.01 \mathrm{~km} \mathrm{~s}^{-1}$.

One additional set of velocities is available for examination. A recomputation of the orbit with the use of the DAO photographic plate velocities (Harper 1932a) results in a center-of-mass velocity of $-20.5 \pm 0.9 \mathrm{~km} \mathrm{~s}^{-1}$. Eliminating two DAO velocities that have very large residuals revises that systemic velocity to $-20.6 \pm 0.6 \mathrm{~km} \mathrm{~s}^{-1}$. Thus, the orbital solutions indicate that the center-of-mass velocity of HR 1528 has a range of $4 \mathrm{~km} \mathrm{~s}^{-1}$. Observatory zero-point velocity differences are usually in the $1-2 \mathrm{~km} \mathrm{~s}^{-1}$ range (e.g., Harper 1932b). Therefore, the difference between the DAO and the more recent data sets suggests that there is a third component in the system. Taken at face value, the velocity change validates the interferometric discovery of

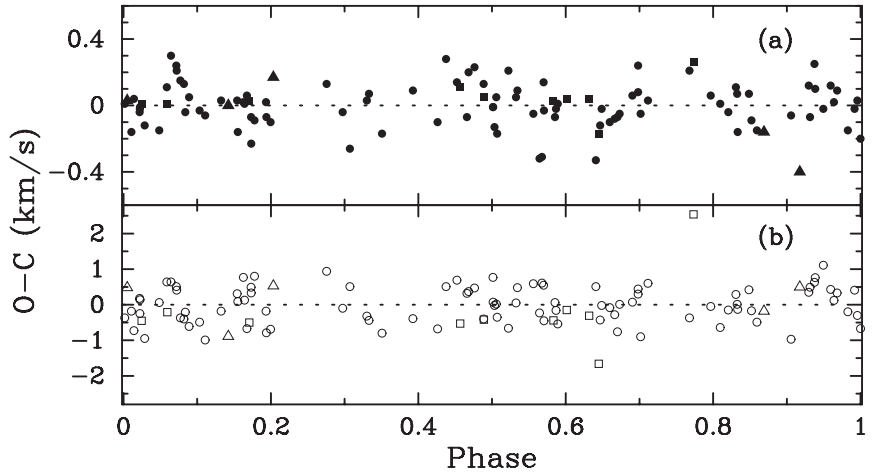

Figure 2. HR 1528 radial velocity residuals vs. orbital phase. Panel (a) shows residuals of the primary, while panel (b) is of the secondary. Circles = Fairborn Observatory, triangles $=\mathrm{KPNO}$, and squares $=\mathrm{McDonald}$ Observatory. Zero phase is a time of maximum velocity of the primary.

a third component (McAlister et al. 1987). However, despite this apparent spectroscopic confirmation, questions remain because over more than a quarter century, there has been no interferometric confirmation of the wider binary. As an additional test of our spectroscopic data, we divided the time span of our Fairborn data in half and computed orbits for the primary velocities of both sets. The two systemic velocities are identical, showing no evidence of orbital motion during the 9.4 yr covered by our new observations.

\subsection{HR 6993}

Between 2002 and 2013 we obtained a total of 119 spectroscopic observations of HR 6993 at three observatories. We began our observing program at McDonald Observatory, acquiring a dozen spectrograms between 2002 and 2005. From 2004 through 2011, we collected 26 observations at KPNO. Finally, from 2004 through 2013, we also observed HR 6993 at Fairborn Observatory, where we obtained 81 usable double-lined spectrograms (Table 4). The number of velocities at each of the three observatories is large enough that we initially analyzed each data set separately, obtaining six orbital solutions, three for the primary and three for the secondary. The variances of the three orbital solutions for the primary resulted in weights of 1.0, 0.4, and 0.2 for the velocities from McDonald, Fairborn, and KPNO, respectively. For the secondary, the weights were $0.2,0.2$, and 0.1 for the same three respective observatories. The center-of-mass velocities of the six data sets agree to within $0.4 \mathrm{~km} \mathrm{~s}^{-1}$. So, we next obtained a simultaneous solution of all our velocities, which produced an orbital period of 14.68463 days. 
Table 4

Radial Velocities of HR 6993

\begin{tabular}{|c|c|c|c|c|c|c|c|c|}
\hline $\begin{array}{l}\text { Hel. Julian Date } \\
\text { (HJD - 2,400,000) }\end{array}$ & Phase & $\begin{array}{c}V_{1} \\
\left(\mathrm{~km} \mathrm{~s}^{-1}\right)\end{array}$ & $\begin{array}{l}(O-C)_{1} \\
\left(\mathrm{~km} \mathrm{~s}^{-1}\right)\end{array}$ & $\mathrm{Wt}_{1}$ & $\begin{array}{c}V_{2} \\
\left(\mathrm{~km} \mathrm{~s}^{-1}\right)\end{array}$ & $\begin{array}{l}(O-C)_{2} \\
\left(\mathrm{~km} \mathrm{~s}^{-1}\right)\end{array}$ & $\mathrm{Wt}_{2}$ & Source $^{a}$ \\
\hline 52392.912 & 0.975 & -25.6 & -0.2 & 1.0 & 48.5 & -0.4 & 0.2 & $\mathrm{McD}$ \\
\hline 52749.948 & 0.289 & 42.9 & -0.5 & 1.0 & -21.1 & 0.0 & 0.2 & $\mathrm{McD}$ \\
\hline 52750.939 & 0.356 & 41.0 & -0.6 & 1.0 & -19.3 & 0.0 & 0.2 & $\mathrm{McD}$ \\
\hline 52888.727 & 0.739 & -7.7 & -0.1 & 1.0 & 30.5 & -0.3 & 0.2 & $\mathrm{McD}$ \\
\hline 52889.698 & 0.805 & -20.2 & -0.2 & 1.0 & 43.5 & 0.2 & 0.2 & $\mathrm{McD}$ \\
\hline 53075.004 & 0.424 & 36.6 & -0.3 & 1.0 & -14.8 & -0.3 & 0.2 & $\mathrm{McD}$ \\
\hline 53110.913 & 0.870 & -30.0 & -0.2 & 1.0 & 53.3 & -0.1 & 0.2 & $\mathrm{McD}$ \\
\hline 53168.840 & 0.815 & -22.0 & -0.4 & 0.2 & 45.2 & 0.2 & 0.1 & KPNO \\
\hline 53169.873 & 0.885 & -31.3 & 0.0 & 0.2 & 54.4 & -0.4 & 0.1 & KPNO \\
\hline 53171.842 & 0.019 & -11.7 & 0.3 & 0.2 & 35.9 & 0.7 & 0.1 & KPNO \\
\hline 53259.633 & 0.997 & -19.4 & -0.1 & 1.0 & 42.6 & 0.0 & 0.2 & $\mathrm{McD}$ \\
\hline 53261.740 & 0.141 & 28.8 & -0.1 & 1.0 & -6.2 & 0.1 & 0.2 & $\mathrm{McD}$ \\
\hline 53276.640 & 0.156 & 32.1 & 0.1 & 0.4 & -8.4 & 1.1 & 0.2 & Fair \\
\hline 53285.683 & 0.771 & -13.2 & 0.4 & 0.4 & 36.9 & 0.0 & 0.2 & Fair \\
\hline 53290.669 & 0.111 & 21.4 & 0.4 & 0.4 & 2.3 & 0.6 & 0.2 & Fair \\
\hline 53302.665 & 0.928 & -31.8 & 0.3 & 0.4 & 55.2 & -0.5 & 0.2 & Fair \\
\hline 53314.629 & 0.743 & -7.9 & 0.3 & 0.4 & 30.7 & -0.7 & 0.2 & Fair \\
\hline 53423.020 & 0.124 & 25.5 & 0.9 & 0.4 & -0.8 & 1.2 & 0.2 & Fair \\
\hline 53470.939 & 0.387 & 39.9 & 0.1 & 0.4 & -17.6 & -0.2 & 0.2 & Fair \\
\hline 53483.831 & 0.265 & 43.1 & 0.0 & 0.4 & -21.3 & -0.5 & 0.2 & Fair \\
\hline 53486.937 & 0.476 & 31.6 & -0.4 & 1.0 & -9.5 & 0.0 & 0.2 & $\mathrm{McD}$ \\
\hline 53489.896 & 0.678 & 3.5 & 0.0 & 1.0 & 19.4 & 0.0 & 0.2 & $\mathrm{McD}$ \\
\hline 53490.952 & 0.750 & -9.8 & -0.2 & 0.2 & 33.0 & 0.2 & 0.1 & KPNO \\
\hline 53496.970 & 0.160 & 33.3 & 0.5 & 0.4 & -9.4 & 0.9 & 0.2 & Fair \\
\hline 53509.940 & 0.043 & -2.6 & 0.4 & 0.4 & 26.0 & -0.1 & 0.2 & Fair \\
\hline 53528.887 & 0.333 & 42.6 & 0.0 & 0.4 & -19.9 & 0.4 & 0.2 & Fair \\
\hline $53536.923^{b}$ & 0.880 & -31.1 & -0.2 & 0.2 & 53.6 & -0.9 & 0.1 & KPNO \\
\hline 53548.906 & 0.696 & 1.1 & 0.8 & 0.4 & 23.5 & 0.8 & 0.2 & Fair \\
\hline 53563.798 & 0.711 & -2.3 & 0.0 & 0.4 & 25.2 & -0.1 & 0.2 & Fair \\
\hline 53608.684 & 0.767 & -12.7 & 0.2 & 0.4 & 35.0 & -1.1 & 0.2 & Fair \\
\hline 53629.741 & 0.201 & 39.1 & 0.1 & 0.4 & -15.9 & 0.7 & 0.2 & Fair \\
\hline $53638.599^{b}$ & 0.804 & -20.3 & -0.5 & 0.2 & 42.8 & -0.3 & 0.1 & KPNO \\
\hline 53663.557 & 0.504 & 28.6 & -0.3 & 1.0 & -7.0 & -0.6 & 0.2 & $\mathrm{McD}$ \\
\hline 53673.638 & 0.190 & 37.6 & -0.1 & 0.4 & -14.6 & 0.7 & 0.2 & Fair \\
\hline 53846.883 & 0.988 & -21.9 & 0.1 & 0.4 & 45.8 & 0.4 & 0.2 & Fair \\
\hline 53851.951 & 0.333 & 41.8 & -0.8 & 0.2 & -20.7 & -0.4 & 0.1 & KPNO \\
\hline 53859.985 & 0.880 & -30.7 & 0.2 & 0.4 & 54.8 & 0.3 & 0.2 & Fair \\
\hline 53872.824 & 0.755 & -10.0 & 0.5 & 0.4 & 33.4 & -0.3 & 0.2 & Fair \\
\hline 53902.910 & 0.804 & -19.5 & 0.1 & 0.4 & 43.2 & 0.2 & 0.2 & Fair \\
\hline 53996.768 & 0.195 & 38.6 & 0.3 & 0.4 & -16.4 & -0.5 & 0.2 & Fair \\
\hline $54005.611^{b}$ & 0.797 & -18.9 & -0.4 & 0.2 & 41.9 & 0.1 & 0.1 & KPNO \\
\hline 54022.646 & 0.957 & -28.9 & 0.1 & 0.4 & 52.6 & 0.1 & 0.2 & Fair \\
\hline $54268.918^{b}$ & 0.728 & -4.8 & 0.7 & 0.2 & 28.7 & 0.1 & 0.1 & KPNO \\
\hline 54269.856 & 0.792 & -18.5 & -1.0 & 0.2 & 39.9 & -0.9 & 0.1 & KPNO \\
\hline 54364.610 & 0.245 & 41.7 & -0.6 & 0.2 & -18.9 & 1.2 & 0.1 & KPNO \\
\hline 54582.961 & 0.114 & 21.2 & -0.6 & 0.2 & 0.6 & -0.2 & 0.1 & KPNO \\
\hline $54586.926^{b}$ & 0.384 & 39.6 & -0.4 & 0.2 & -18.1 & -0.5 & 0.1 & KPNO \\
\hline 54643.882 & 0.263 & 42.4 & -0.6 & 0.2 & -21.0 & -0.3 & 0.1 & KPNO \\
\hline 54947.966 & 0.970 & -27.1 & -0.6 & 0.2 & 48.8 & -1.2 & 0.1 & KPNO \\
\hline 54950.941 & 0.173 & 34.1 & -1.0 & 0.2 & -12.7 & 0.0 & 0.1 & KPNO \\
\hline 55006.916 & 0.985 & -22.0 & 1.0 & 0.2 & 47.6 & 1.2 & 0.1 & KPNO \\
\hline 55094.670 & 0.960 & -27.3 & 1.1 & 0.2 & 52.7 & 0.8 & 0.1 & KPNO \\
\hline 55246.010 & 0.266 & 43.3 & 0.2 & 0.4 & -20.5 & 0.3 & 0.2 & Fair \\
\hline 55274.896 & 0.234 & 41.8 & 0.1 & 0.4 & -19.5 & -0.1 & 0.2 & Fair \\
\hline 55284.004 & 0.854 & -27.5 & 0.3 & 0.4 & 51.2 & -0.1 & 0.2 & Fair \\
\hline 55293.881 & 0.526 & 26.0 & -0.2 & 0.4 & -2.6 & 1.0 & 0.2 & Fair \\
\hline 55312.935 & 0.824 & -23.2 & 0.0 & 0.4 & 47.1 & 0.5 & 0.2 & Fair \\
\hline 55312.953 & 0.825 & -23.9 & -0.5 & 0.2 & 46.0 & -0.8 & 0.1 & KPNO \\
\hline 55314.985 & 0.964 & -28.6 & -0.7 & 0.2 & 49.9 & -1.5 & 0.1 & KPNO \\
\hline 55320.808 & 0.360 & 42.2 & 0.8 & 0.4 & -18.7 & 0.4 & 0.2 & Fair \\
\hline 55329.965 & 0.984 & -23.1 & 0.1 & 0.4 & 47.2 & 0.5 & 0.2 & Fair \\
\hline 55341.931 & 0.799 & -18.5 & 0.2 & 0.4 & 42.3 & 0.2 & 0.2 & Fair \\
\hline 55370.920 & 0.773 & -13.4 & 0.5 & 0.2 & 37.0 & -0.1 & 0.1 & KPNO \\
\hline 55376.940 & 0.183 & 36.5 & -0.1 & 0.4 & -13.8 & 0.4 & 0.2 & Fair \\
\hline
\end{tabular}


Table 4

(Continued)

\begin{tabular}{|c|c|c|c|c|c|c|c|c|}
\hline $\begin{array}{l}\text { Hel. Julian Date } \\
\text { (HJD - 2,400,000) }\end{array}$ & Phase & $\begin{array}{c}V_{1} \\
\left(\mathrm{~km} \mathrm{~s}^{-1}\right)\end{array}$ & $\begin{array}{c}(O-C)_{1} \\
\left(\mathrm{~km} \mathrm{~s}^{-1}\right)\end{array}$ & $\mathrm{Wt}_{1}$ & $\begin{array}{c}V_{2} \\
\left(\mathrm{~km} \mathrm{~s}^{-1}\right)\end{array}$ & $\begin{array}{c}(O-C)_{2} \\
\left(\mathrm{~km} \mathrm{~s}^{-1}\right)\end{array}$ & $\mathrm{Wt}_{2}$ & Source $^{a}$ \\
\hline 55385.873 & 0.791 & -17.3 & 0.0 & 0.4 & 39.7 & -0.9 & 0.2 & Fair \\
\hline 55451.681 & 0.272 & 43.9 & 0.7 & 0.4 & -20.4 & 0.5 & 0.2 & Fair \\
\hline 55458.682 & 0.749 & -9.0 & 0.4 & 0.4 & 32.8 & 0.2 & 0.2 & Fair \\
\hline 55469.648 & 0.496 & 29.8 & 0.0 & 0.4 & -6.6 & 0.7 & 0.2 & Fair \\
\hline 55477.652 & 0.041 & -2.9 & 0.9 & 0.4 & 27.1 & 0.2 & 0.2 & Fair \\
\hline 55488.645 & 0.790 & -16.8 & 0.2 & 0.4 & 40.6 & 0.2 & 0.2 & Fair \\
\hline 55623.948 & 0.003 & -17.3 & 0.1 & 0.4 & 41.5 & 0.8 & 0.2 & Fair \\
\hline 55635.004 & 0.756 & -10.3 & 0.5 & 0.4 & 34.2 & 0.2 & 0.2 & Fair \\
\hline 55643.894 & 0.362 & 42.0 & 0.7 & 0.4 & -19.1 & -0.1 & 0.2 & Fair \\
\hline 55649.896 & 0.770 & -13.4 & 0.1 & 0.4 & 37.2 & 0.5 & 0.2 & Fair \\
\hline 55656.951 & 0.251 & 43.1 & 0.5 & 0.4 & -20.0 & 0.3 & 0.2 & Fair \\
\hline 55664.841 & 0.788 & -17.2 & -0.4 & 0.4 & 40.1 & 0.0 & 0.2 & Fair \\
\hline 55670.861 & 0.198 & 38.6 & 0.0 & 0.4 & -15.7 & 0.6 & 0.2 & Fair \\
\hline 55674.927 & 0.475 & 32.3 & 0.2 & 0.4 & -10.1 & -0.5 & 0.2 & Fair \\
\hline 55678.821 & 0.740 & -8.0 & -0.2 & 0.4 & 30.8 & -0.1 & 0.2 & Fair \\
\hline 55679.926 & 0.815 & -22.3 & -0.6 & 0.2 & 43.7 & -1.4 & 0.1 & KPNO \\
\hline 55680.944 & 0.885 & -32.2 & -0.9 & 0.2 & 54.1 & -0.7 & 0.1 & KPNO \\
\hline 55681.934 & 0.952 & -30.6 & -0.8 & 0.2 & 52.6 & -0.7 & 0.1 & KPNO \\
\hline 55684.861 & 0.152 & 31.8 & 0.6 & 0.4 & -8.3 & 0.4 & 0.2 & Fair \\
\hline 55688.803 & 0.420 & 37.7 & 0.4 & 0.4 & -15.0 & -0.1 & 0.2 & Fair \\
\hline 55694.800 & 0.828 & -24.2 & -0.3 & 0.4 & 47.9 & 0.5 & 0.2 & Fair \\
\hline 55699.784 & 0.168 & 34.5 & 0.2 & 0.4 & -11.9 & -0.1 & 0.2 & Fair \\
\hline 55716.737 & 0.322 & 42.9 & 0.0 & 0.4 & -20.9 & -0.3 & 0.2 & Fair \\
\hline 55724.954 & 0.882 & -31.1 & -0.1 & 0.4 & 54.2 & -0.4 & 0.2 & Fair \\
\hline 55730.810 & 0.281 & 42.8 & -0.5 & 0.2 & -21.4 & -0.3 & 0.1 & KPNO \\
\hline 55732.850 & 0.419 & 36.6 & -0.7 & 0.2 & -15.5 & -0.5 & 0.1 & KPNO \\
\hline 55743.861 & 0.169 & 34.2 & -0.3 & 0.4 & -12.7 & -0.6 & 0.2 & Fair \\
\hline 55846.666 & 0.170 & 34.2 & -0.5 & 0.4 & -11.6 & 0.6 & 0.2 & Fair \\
\hline 55954.036 & 0.482 & 32.0 & 0.6 & 0.4 & -8.7 & 0.2 & 0.2 & Fair \\
\hline 55976.012 & 0.978 & -24.8 & -0.2 & 0.4 & 48.7 & 0.7 & 0.2 & Fair \\
\hline 55993.002 & 0.135 & 27.2 & -0.4 & 0.4 & -5.3 & -0.3 & 0.2 & Fair \\
\hline 56011.977 & 0.428 & 37.2 & 0.5 & 0.4 & -14.6 & -0.3 & 0.2 & Fair \\
\hline 56017.951 & 0.834 & -24.8 & 0.1 & 0.4 & 48.7 & 0.3 & 0.2 & Fair \\
\hline 56046.784 & 0.798 & -19.2 & -0.6 & 0.4 & 42.8 & 0.9 & 0.2 & Fair \\
\hline 56055.979 & 0.424 & 37.1 & 0.1 & 0.4 & -15.3 & -0.7 & 0.2 & Fair \\
\hline 56061.833 & 0.823 & -23.3 & -0.3 & 0.4 & 47.0 & 0.6 & 0.2 & Fair \\
\hline 56062.796 & 0.888 & -31.6 & -0.1 & 0.4 & 55.0 & -0.1 & 0.2 & Fair \\
\hline 56063.798 & 0.957 & -29.2 & -0.1 & 0.4 & 52.2 & -0.4 & 0.2 & Fair \\
\hline 56067.774 & 0.227 & 41.7 & 0.4 & 0.4 & -20.1 & -1.1 & 0.2 & Fair \\
\hline 56074.766 & 0.703 & -0.5 & 0.5 & 0.4 & 24.7 & 0.7 & 0.2 & Fair \\
\hline 56077.773 & 0.908 & -32.2 & 0.2 & 0.4 & 55.2 & -0.8 & 0.2 & Fair \\
\hline 56082.756 & 0.248 & 42.6 & 0.1 & 0.4 & -20.6 & -0.4 & 0.2 & Fair \\
\hline 56095.912 & 0.143 & 29.8 & 0.4 & 0.4 & -7.4 & -0.5 & 0.2 & Fair \\
\hline 56100.736 & 0.472 & 32.8 & 0.4 & 0.4 & -10.0 & 0.0 & 0.2 & Fair \\
\hline 56126.912 & 0.254 & 41.9 & -0.9 & 0.4 & -20.8 & -0.3 & 0.2 & Fair \\
\hline 56213.655 & 0.162 & 32.6 & -0.5 & 0.4 & -10.4 & 0.3 & 0.2 & Fair \\
\hline 56228.642 & 0.182 & 36.1 & -0.4 & 0.4 & -14.8 & -0.7 & 0.2 & Fair \\
\hline 56347.017 & 0.243 & 42.1 & -0.2 & 0.4 & -20.8 & -0.8 & 0.2 & Fair \\
\hline 56389.900 & 0.164 & 33.7 & 0.2 & 0.4 & -11.6 & -0.5 & 0.2 & Fair \\
\hline 56400.853 & 0.909 & -32.0 & 0.4 & 0.4 & 55.9 & -0.1 & 0.2 & Fair \\
\hline 56405.845 & 0.249 & 42.9 & 0.3 & 0.4 & -21.6 & -1.3 & 0.2 & Fair \\
\hline 56406.863 & 0.319 & 43.6 & 0.6 & 0.4 & -21.8 & -1.1 & 0.2 & Fair \\
\hline 56409.819 & 0.520 & 27.3 & 0.3 & 0.4 & -4.1 & 0.3 & 0.2 & Fair \\
\hline 56414.835 & 0.862 & -28.8 & 0.0 & 0.4 & 51.7 & -0.7 & 0.2 & Fair \\
\hline 56419.797 & 0.199 & 39.2 & 0.4 & 0.4 & -17.0 & -0.6 & 0.2 & Fair \\
\hline
\end{tabular}

Notes.

${ }^{\mathrm{a}} \mathrm{McD}=\mathrm{McDonald}$ Observatory, KPNO = Kitt Peak National Observatory, Fair = Fairborn Observatory.

${ }^{\mathrm{b}}$ Blue wavelength spectrum.

The only other extensive velocity data set is that of Petrie (1948), who, over the course of just under 12 months, obtained at the DAO 17 double-lined spectrograms with a linear dispersion of $11 \AA \mathrm{mm}^{-1}$ at $\mathrm{H} \gamma$. Despite this relatively high dispersion and the narrow lines of the two components, an average of just six lines per component was measured on each plate. The precision of the better primary velocities is very poor, with a solution of those velocities producing relative weights of less 


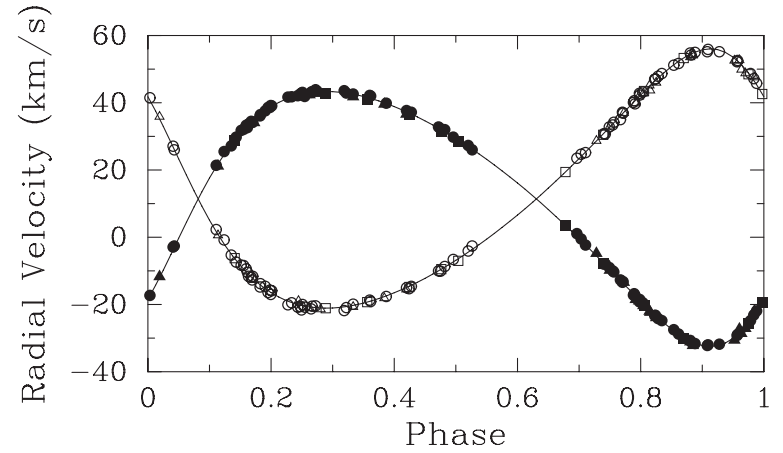

Figure 3. Radial velocities of HR 6993 compared with the computed velocity curves. Filled and open symbols represent the primary and secondary, respectively. Circles $=$ Fairborn Observatory, triangles $=\mathrm{KPNO}$, and squares $=$ McDonald Observatory. Zero phase is a time of periastron passage.

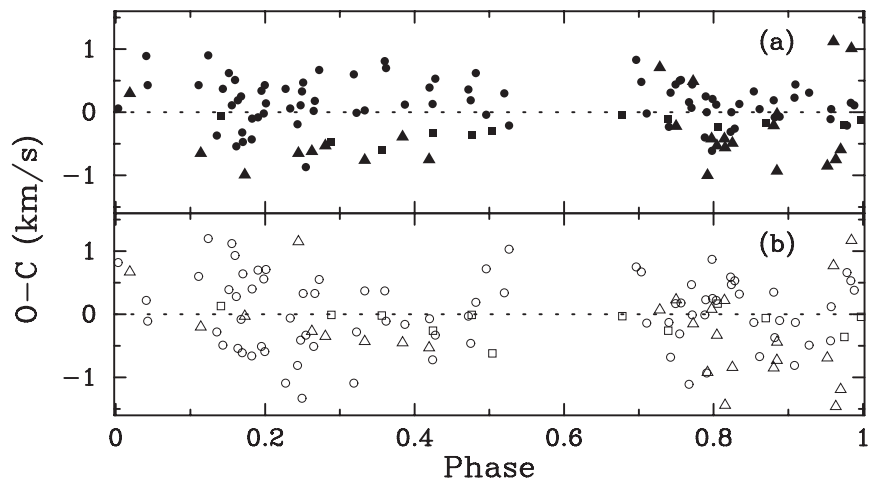

Figure 4. HR 6993 radial velocity residuals vs. orbital phase. Panel (a) shows residuals of the primary, while panel (b) is of the secondary. Circles = Fairborn Observatory, triangles $=\mathrm{KPNO}$, and squares = McDonald Observatory. Zero phase is a time of periastron passage.

than 0.01. Thus, we have not included the DAO velocities in our solution.

The new orbital elements from our double-lined solution are given in Table 5. Our primary and secondary velocities are compared with the computed velocity curves in Figure 3. In that plot, zero phase is a time of periastron passage. Figure 4 shows the velocity residuals versus phase for the primary and secondary. Although the magnitude difference between the two components is modest (Section 5.2), the typical residual of the secondary is somewhat greater than that of the primary.

For comparison, we also list in Table 5 the orbital elements of Petrie (1948). They are in general agreement with our much more precise results. However, our eccentricity is $18 \%$ larger, and we find that the stronger lined star, which Petrie called the primary, is as expected the slightly more massive one, which is the reverse of Petrie's result.

\subsection{Sge}

Our observation of 2 Sge was begun at McDonald Observatory where we acquired 12 spectrograms between 2002 and 2005. From 2004 through 2012, we obtained 28 additional observations at KPNO. Our most extensive observing campaign was conducted at Fairborn Observatory. There between 2004 and 2013 we collected 85 usable spectrograms. A KPNO spectrum of 2 Sge in the $4500 \AA$ region is shown in Figure 5. Clearly visible is the significant line strength difference between the short-period primary and secondary components. A KPNO red wavelength spectrum is presented in Figure 6 showing the weakness of the A-star lines in the $6430 \AA$ region. Also seen are very

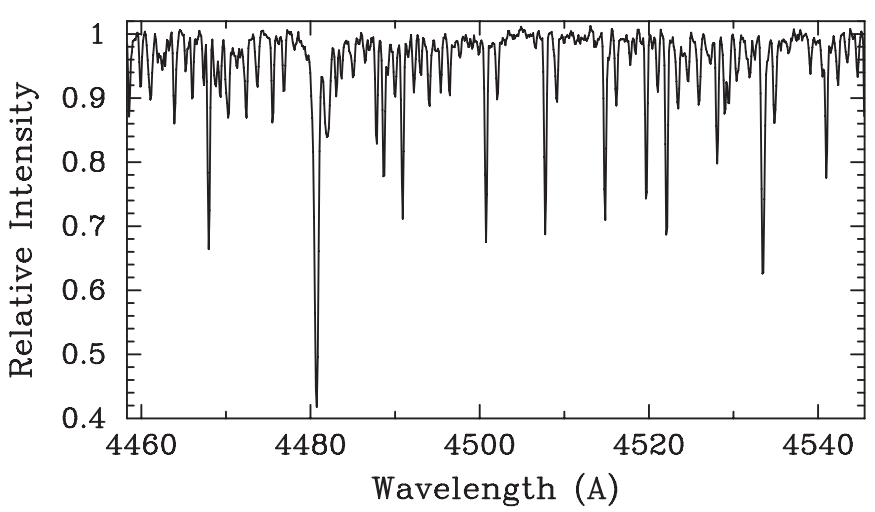

Figure 5. KPNO spectrum of the $4500 \AA$ region of 2 Sge. It shows the narrowlined nature of both short-period components as well as the large line strength difference between the strong short-period primary features and the much weaker secondary features. The strongest line in this region is the Mg II line at $4481 \AA$. Most of the other features are Fe II or Ti II lines.

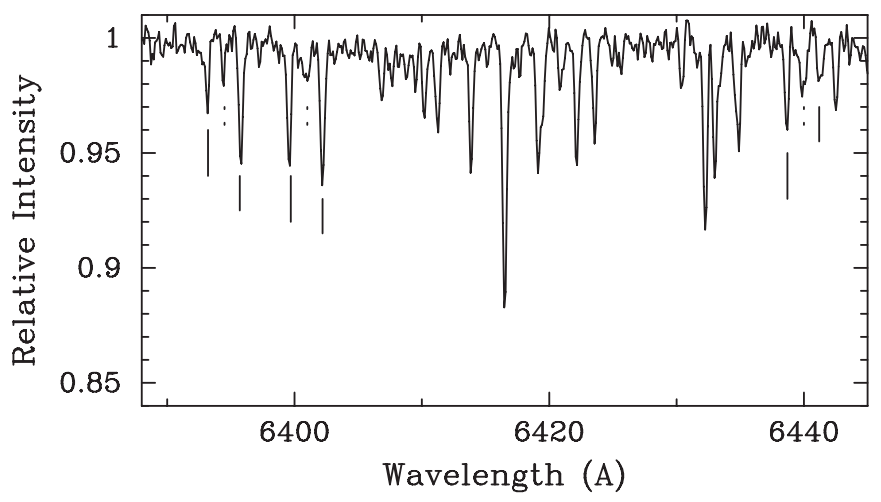

Figure 6. KPNO spectrum of the $6420 \AA$ region of 2 Sge. Three sets of triple lines are indicated. Solid lines identify the short-period primary (longer line) and secondary (shorter line) components, while very weak features of the close visual component are identified by dashed lines. In this region for $\mathrm{Fe}_{\mathrm{I}}$ lines it is the secondary that is the stronger component. The triplet near $6440 \AA$ is that of a Ca I line.

weak lines of the close visual companion that was discovered by the Hipparcos team (Perryman et al. 1997). Our new velocities of the short-period binary as well as those of the faint, close visual companion are given in Table 6.

The number of velocities of 2 Sge at each of the three observatories is large enough, and the orbital phase coverage is good enough so that just as we did with HR 6993, we initially analyzed each data set separately, obtaining six separate orbital solutions, three for the primary and three for the secondary. The eccentricity of each solution was very small, ranging from 0.004 to 0.011 . However, the longitude of periastron values of the primary were similar and the values for the secondary differed by roughly $180^{\circ}$ from those of the primary, indicating that the short-period orbit of 2 Sge does indeed have a very small eccentricity. Velocity residuals from the Fairborn orbit of the short-period secondary suggest that there might be a small $+0.3 \mathrm{~km} \mathrm{~s}^{-1}$ shift in its center-of-mass velocity over the course of our observations, but that result was not confirmed in the other data sets. The center-of-mass velocities of the six solutions have a range of $0.6 \mathrm{~km} \mathrm{~s}^{-1}$, and so we have chosen to combine all our velocities into a single simultaneous solution. From the six individual solutions the weights for the short-period primary are 1.0, 0.3, and 0.3 for the velocities from McDonald, KPNO, and Fairborn, respectively. For the secondary the weights are $1.0,0.9$, and 0.4 for the same three observatories. The 
Table 5

Orbital Elements and Related Parameters of HR 6993

\begin{tabular}{lcc}
\hline \hline Parameter & Petrie $(1948)$ & This Study \\
\hline$P$ (days) & $14.674 \pm 0.007$ & $14.684636 \pm 0.000029$ \\
$T$ (HJD) & 2432304.628 & $2454405.073 \pm 0.011$ \\
$e$ & $0.211 \pm 0.012$ & $0.2495 \pm 0.0011$ \\
$\omega(\mathrm{deg})$ & $224.9 \pm 3.8$ & $230.90 \pm 0.30$ \\
$K_{1}\left(\mathrm{~km} \mathrm{~s}^{-1}\right)$ & $38.56 \pm 0.53$ & $37.918 \pm 0.050$ \\
$K_{2}\left(\mathrm{~km} \mathrm{~s}^{-1}\right)$ & $38.07 \pm 0.56$ & $38.569 \pm 0.076$ \\
$\gamma\left(\mathrm{km} \mathrm{s}^{-1}\right)$ & $11.39 \pm 0.35$ & $11.415 \pm 0.031$ \\
$m_{1} \sin ^{3} i\left(M_{\odot}\right)$ & 0.320 & $0.3118 \pm 0.0012$ \\
$m_{2} \sin ^{3} i\left(M_{\odot}\right)$ & 0.324 & $0.3065 \pm 0.0010$ \\
$a_{1} \sin \left(10^{6} \mathrm{~km}\right)$ & 7.621 & $7.414 \pm 0.010$ \\
$a_{2} \sin i\left(10^{6} \mathrm{~km}\right)$ & 7.522 & $7.542 \pm 0.015$ \\
Standard error of an unit weight observation $\left(\mathrm{km} \mathrm{s}^{-1}\right)$ & $\ldots$ & 0.25 \\
\hline
\end{tabular}

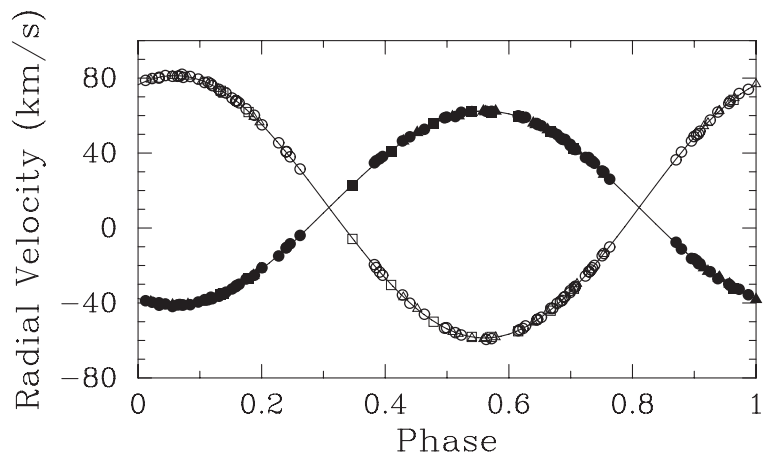

Figure 7. Radial velocities of 2 Sge compared with the computed velocity curves. Filled and open symbols represent the primary and secondary, respectively. Circles $=$ Fairborn Observatory, triangles $=\mathrm{KPNO}$, and squares $=\mathrm{Mc}-$ Donald Observatory. Zero phase is a time of periastron passage.

higher weights assigned to the KPNO and Fairborn secondary velocities reflect the fact that at red wavelengths the neutral Fe lines of the secondary are stronger than those of the primary.

The only other major velocity data set consists of the old photographic plate results from the Dominion Observatory in Ottawa (Young 1917). An orbital solution of those primary velocities produces a standard deviation per plate of $6.2 \mathrm{~km} \mathrm{~s}^{-1}$ resulting in such low weights for those velocities that they do not significantly improve the value of the period. Thus, the new orbital elements from our double-lined solution are given in Table 7. Our primary and secondary velocities are compared with the computed velocity curves in Figure 7. In that plot zero phase is a time of periastron passage. As seen in Figure 8, the stronger secondary lines in the KPNO and Fairborn spectra result in smaller velocity residuals for the secondary.

The orbital elements for 2 Sge, computed by Luyten (1936) from the Dominion Observatory velocities, are shown for comparison in Table 7. Given that the original solution of the Dominion Observatory velocities was described as preliminary (Young 1917), the circular orbital elements from those velocities are in reasonable agreement with our much more precise results.

Velocities of the faint, close visual secondary, discovered by the Hipparcos team (Perryman et al. 1997), are measurable if the spectrograms have a high enough signal-to-noise ratio. Those velocities (Table 6) are shown in a two-panel plot. In Figure 9, panel (a) presents the Fairborn velocities plotted versus Julian Date. When divided into three subsets, the one centered near

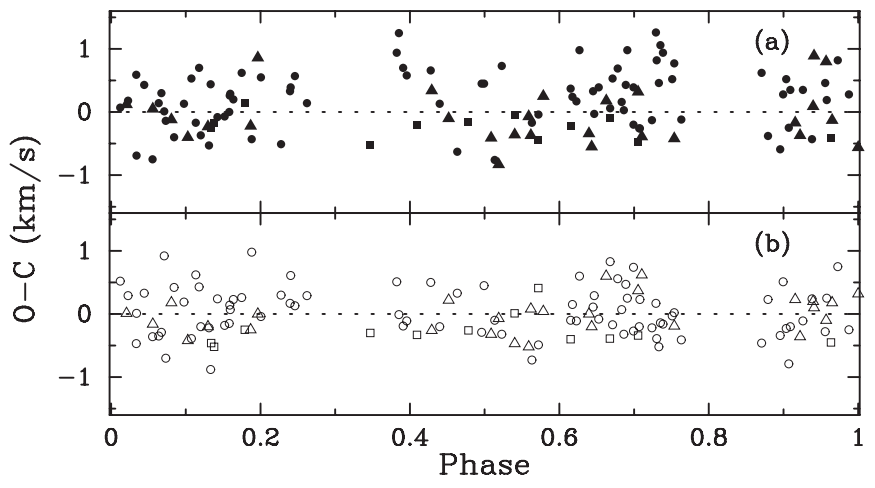

Figure 8. 2 Sge radial velocity residuals vs. orbital phase. Panel (a) shows residuals of the primary, while panel (b) is of the secondary. Circles = Fairborn Observatory, triangles $=\mathrm{KPNO}$, and squares $=\mathrm{McDonald}$ Observatory. Zero phase is a time of periastron passage.

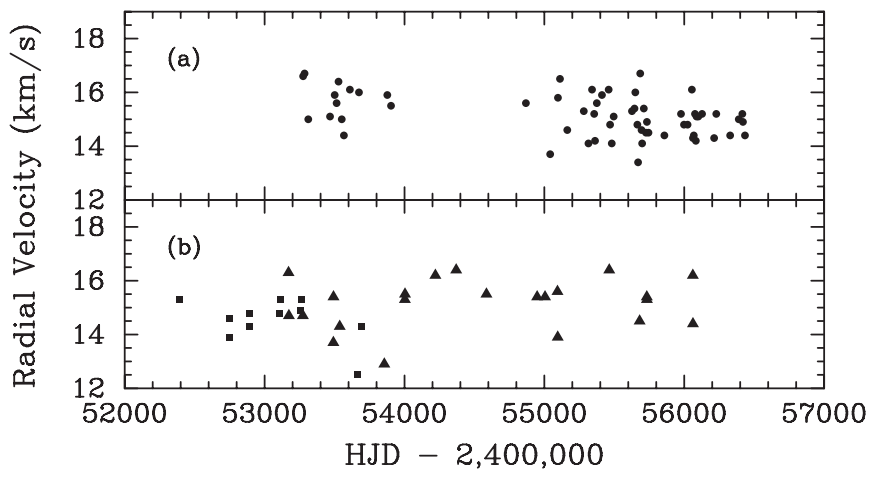

Figure 9. Radial velocities of the close visual secondary of 2 Sge. Panel (a) plots the Fairborn Observatory radial velocities vs. Julian Date. Those velocities indicate a slight negative trend over time. Panel (b) presents the McDonald Observatory (solid squares) and KPNO (solid triangles) velocities, which do not confirm the trend suggested by the Fairborn velocities.

JD 2,453,500 has an average velocity of $15.7 \mathrm{~km} \mathrm{~s}^{-1}$ while the subset centered near JD 2,456,200 has an average of $14.9 \mathrm{~km} \mathrm{~s}^{-1}$. Thus, these data indicate a velocity decrease of $0.8 \mathrm{~km} \mathrm{~s}^{-1}$ over the nearly $9 \mathrm{yr}$ span of observation. Seeming to suggest possible orbital motion, this change is in the opposite direction to the $0.3 \mathrm{~km} \mathrm{~s}^{-1}$ shift found in the Fairborn velocity residuals of the short-period secondary, which was mentioned previously. Panel (b) plots our other two data sets, the McDonald and KPNO velocities. These velocities show no evidence of the change suggested by the Fairborn velocities. Thus, we conclude that any velocity change of the short-period binary center of mass and that of its close visual secondary remains to be confirmed. 
Table 6

Radial Velocities of 2 Sge

\begin{tabular}{|c|c|c|c|c|c|c|c|c|c|}
\hline $\begin{array}{l}\text { Hel. Julian Date } \\
(\mathrm{HJD}-2,400,000)\end{array}$ & Phase & $\begin{array}{c}V_{1} \\
\left(\mathrm{~km} \mathrm{~s}^{-1}\right)\end{array}$ & $\begin{array}{c}(O-C)_{1} \\
\left(\mathrm{~km} \mathrm{~s}^{-1}\right)\end{array}$ & $\mathrm{Wt}_{1}$ & $\begin{array}{c}V_{2} \\
\left(\mathrm{~km} \mathrm{~s}^{-1}\right)\end{array}$ & $\begin{array}{c}(O-C)_{2} \\
\left(\mathrm{~km} \mathrm{~s}^{-1}\right)\end{array}$ & $\mathrm{Wt}_{2}$ & $\begin{array}{c}V_{3} \\
\left(\mathrm{~km} \mathrm{~s}^{-1}\right)\end{array}$ & $\overline{\text { Source }^{\mathrm{a}}}$ \\
\hline 52391.934 & 0.134 & -35.7 & -0.3 & 1.0 & 73.2 & -0.5 & 1.0 & 15.3 & $\mathrm{McD}$ \\
\hline 52749.968 & 0.572 & 61.9 & -0.4 & 1.0 & -58.1 & 0.4 & 1.0 & 13.9 & $\mathrm{McD}$ \\
\hline 52750.954 & 0.705 & 42.3 & -0.5 & 1.0 & -32.4 & -0.3 & 1.0 & 14.6 & $\mathrm{McD}$ \\
\hline 52888.745 & 0.347 & 22.6 & -0.5 & 1.0 & -5.8 & -0.3 & 1.0 & $\ldots$ & $\mathrm{McD}$ \\
\hline 52889.716 & 0.478 & 55.7 & -0.2 & 1.0 & -50.0 & -0.3 & 1.0 & 14.8 & $\mathrm{McD}$ \\
\hline 52890.727 & 0.615 & 59.3 & -0.2 & 1.0 & -55.1 & -0.4 & 1.0 & 14.3 & $\mathrm{McD}$ \\
\hline 53108.947 & 0.138 & -35.0 & -0.2 & 1.0 & 72.3 & -0.5 & 1.0 & 14.8 & $\mathrm{McD}$ \\
\hline 53110.955 & 0.410 & 41.1 & -0.2 & 1.0 & -30.4 & -0.3 & 1.0 & 15.3 & $\mathrm{McD}$ \\
\hline 53169.908 & 0.385 & 35.9 & 1.2 & 0.3 & -21.1 & 0.0 & 0.4 & $\ldots$ & Fair \\
\hline 53170.895 & 0.519 & 59.9 & -0.8 & 0.3 & -56.4 & -0.1 & 0.9 & 16.3 & KPNO \\
\hline 53173.877 & 0.922 & -23.1 & -0.4 & 0.3 & 56.1 & -0.4 & 0.9 & 14.7 & KPNO \\
\hline 53259.751 & 0.540 & 62.0 & 0.0 & 1.0 & -58.1 & 0.0 & 1.0 & 14.9 & $\mathrm{McD}$ \\
\hline 53260.694 & 0.668 & 51.3 & -0.1 & 1.0 & -44.1 & -0.4 & 1.0 & 15.3 & $\mathrm{McD}$ \\
\hline 53273.715 & 0.429 & 46.5 & 0.3 & 0.3 & -36.9 & -0.3 & 0.9 & 14.7 & KPNO \\
\hline 53275.774 & 0.708 & 41.9 & -0.3 & 0.3 & -31.0 & 0.2 & 0.4 & 16.6 & Fair \\
\hline 53285.737 & 0.056 & -41.9 & -0.7 & 0.3 & 81.0 & -0.4 & 0.4 & 16.7 & Fair \\
\hline 53290.723 & 0.730 & 36.9 & 0.8 & 0.3 & -23.4 & -0.4 & 0.4 & $\ldots$ & Fair \\
\hline 53311.664 & 0.563 & 62.3 & -0.2 & 0.3 & -59.4 & -0.7 & 0.4 & $\ldots$ & Fair \\
\hline 53312.669 & 0.699 & 44.1 & -0.2 & 0.3 & -34.4 & -0.3 & 0.4 & 15.0 & Fair \\
\hline 53467.892 & 0.699 & 44.7 & 0.4 & 0.3 & -33.4 & 0.7 & 0.4 & 15.1 & Fair \\
\hline 53491.972 & 0.957 & -29.8 & 0.8 & 0.3 & 67.0 & -0.1 & 0.9 & 13.7 & KPNO \\
\hline 53492.887 & 0.081 & -40.8 & -0.1 & 0.3 & 80.9 & 0.2 & 0.9 & 15.4 & KPNO \\
\hline 53500.850 & 0.158 & -31.3 & 0.0 & 0.3 & 67.9 & -0.1 & 0.4 & 15.9 & Fair \\
\hline 53514.962 & 0.067 & -40.8 & 0.3 & 0.3 & 81.0 & -0.3 & 0.4 & 15.6 & Fair \\
\hline 53528.921 & 0.956 & -29.9 & 0.5 & 0.3 & 66.5 & -0.3 & 0.4 & 16.4 & Fair \\
\hline 53536.792 & 0.021 & -39.5 & 0.1 & 0.3 & 79.3 & 0.0 & 0.9 & 14.3 & KPNO \\
\hline 53551.964 & 0.073 & -41.1 & -0.1 & 0.3 & 80.4 & -0.7 & 0.4 & 15.0 & Fair \\
\hline 53609.868 & 0.907 & -19.0 & -0.2 & 0.3 & 50.3 & -0.8 & 0.4 & 16.1 & Fair \\
\hline 53630.798 & 0.739 & 34.6 & 0.9 & 0.3 & -19.9 & -0.2 & 0.4 & $\ldots$ & Fair \\
\hline 53646.742 & 0.896 & -16.2 & -0.6 & 0.3 & 46.5 & -0.3 & 0.4 & $\ldots$ & Fair \\
\hline 53663.619 & 0.179 & -26.8 & 0.1 & 1.0 & 61.9 & -0.3 & 1.0 & 12.5 & $\mathrm{McD}$ \\
\hline 53674.649 & 0.671 & 51.2 & 0.5 & 0.3 & -42.9 & -0.2 & 0.4 & 16.0 & Fair \\
\hline 53691.594 & 0.964 & -32.3 & -0.4 & 1.0 & 68.4 & -0.5 & 1.0 & 14.3 & $\mathrm{McD}$ \\
\hline 53855.930 & 0.196 & -21.9 & 0.9 & 0.3 & 56.5 & 0.0 & 0.9 & 12.9 & KPNO \\
\hline 53863.807 & 0.262 & -3.9 & 0.1 & 0.3 & 31.5 & 0.3 & 0.4 & $\ldots$ & Fair \\
\hline 53876.980 & 0.044 & -40.5 & 0.4 & 0.3 & 81.4 & 0.3 & 0.4 & 15.9 & Fair \\
\hline 53903.900 & 0.686 & 47.4 & 0.0 & 0.3 & -38.6 & -0.3 & 0.4 & 15.5 & Fair \\
\hline 54002.722 & 0.056 & -41.1 & 0.1 & 0.3 & 81.2 & -0.2 & 0.9 & 15.3 & KPNO \\
\hline 54003.691 & 0.187 & -25.3 & -0.2 & 0.3 & 59.4 & -0.2 & 0.9 & 15.5 & KPNO \\
\hline $54005.649^{b}$ & 0.452 & 51.0 & -0.1 & 0.3 & -43.1 & 0.2 & 0.9 & $\ldots$ & KPNO \\
\hline 54022.734 & 0.763 & 26.1 & -0.1 & 0.3 & -10.1 & -0.4 & 0.4 & $\ldots$ & Fair \\
\hline 54220.942 & 0.579 & 62.4 & 0.3 & 0.3 & -58.2 & 0.0 & 0.9 & 16.2 & KPNO \\
\hline $54368.628^{b}$ & 0.559 & 62.4 & -0.1 & 0.3 & -59.2 & -0.5 & 0.9 & $\ldots$ & KPNO \\
\hline 54369.710 & 0.705 & 43.1 & 0.3 & 0.3 & -31.7 & 0.4 & 0.9 & 16.4 & KPNO \\
\hline 54583.006 & 0.562 & 62.1 & -0.4 & 0.3 & -58.6 & 0.1 & 0.9 & $\ldots$ & KPNO \\
\hline 54585.986 & 0.965 & -32.3 & -0.1 & 0.3 & 69.4 & 0.2 & 0.9 & 15.5 & KPNO \\
\hline $54644.940^{\mathrm{b}}$ & 0.941 & -26.3 & 0.9 & 0.3 & 62.6 & 0.1 & 0.9 & $\ldots$ & KPNO \\
\hline 54868.012 & 0.120 & -37.7 & -0.4 & 0.3 & 76.0 & -0.2 & 0.4 & 15.6 & Fair \\
\hline 54947.985 & 0.940 & -26.8 & 0.1 & 0.3 & 62.3 & 0.2 & 0.9 & 15.4 & KPNO \\
\hline 55004.900 & 0.640 & 56.0 & -0.3 & 0.3 & -50.4 & 0.0 & 0.9 & 15.4 & KPNO \\
\hline 55041.954 & 0.653 & 54.6 & 0.4 & 0.3 & -47.6 & -0.1 & 0.4 & 13.7 & Fair \\
\hline 55043.779 & 0.899 & -16.4 & 0.3 & 0.3 & 48.8 & 0.5 & 0.4 & $\ldots$ & Fair \\
\hline 55044.774 & 0.034 & -41.2 & -0.7 & 0.3 & 80.5 & 0.0 & 0.4 & $\ldots$ & Fair \\
\hline 55071.789 & 0.689 & 47.2 & 0.4 & 0.3 & -37.0 & 0.5 & 0.4 & $\ldots$ & Fair \\
\hline 55093.771 & 0.663 & 52.5 & 0.2 & 0.3 & -44.4 & 0.6 & 0.9 & 15.6 & KPNO \\
\hline 55095.639 & 0.916 & -21.2 & -0.2 & 0.3 & 54.4 & 0.2 & 0.9 & 13.9 & KPNO \\
\hline 55096.789 & 0.071 & -41.0 & 0.0 & 0.3 & 82.1 & 0.9 & 0.4 & 15.8 & Fair \\
\hline 55111.670 & 0.084 & -40.9 & -0.4 & 0.3 & 80.9 & 0.4 & 0.4 & 16.5 & Fair \\
\hline 55138.640 & 0.733 & 35.7 & 0.5 & 0.3 & -22.4 & -0.5 & 0.4 & $\ldots$ & Fair \\
\hline 55163.625 & 0.113 & -38.3 & -0.2 & 0.3 & 77.9 & 0.6 & 0.4 & 14.6 & Fair \\
\hline 55281.924 & 0.118 & -36.9 & 0.7 & 0.3 & 77.0 & 0.4 & 0.4 & 15.3 & Fair \\
\hline 55304.910 & 0.228 & -14.9 & -0.5 & 0.3 & 45.5 & 0.3 & 0.4 & $\ldots$ & Fair \\
\hline 55314.847 & 0.572 & 62.3 & 0.0 & 0.3 & -59.0 & -0.5 & 0.4 & 14.1 & Fair \\
\hline 55320.936 & 0.396 & 38.2 & 0.6 & 0.3 & -25.2 & -0.1 & 0.4 & $\ldots$ & Fair \\
\hline
\end{tabular}


Table 6

(Continued)

\begin{tabular}{|c|c|c|c|c|c|c|c|c|c|}
\hline $\begin{array}{l}\text { Hel. Julian Date } \\
\text { (HJD - 2,400,000) }\end{array}$ & Phase & $\begin{array}{c}V_{1} \\
\left(\mathrm{~km} \mathrm{~s}^{-1}\right)\end{array}$ & $\begin{array}{l}(O-C)_{1} \\
\left(\mathrm{~km} \mathrm{~s}^{-1}\right)\end{array}$ & $\mathrm{Wt}_{1}$ & $\begin{array}{c}V_{2} \\
\left(\mathrm{~km} \mathrm{~s}^{-1}\right)\end{array}$ & $\begin{array}{c}(O-C)_{2} \\
\left(\mathrm{~km} \mathrm{~s}^{-1}\right)\end{array}$ & $\mathrm{Wt}_{2}$ & $\begin{array}{c}V_{3} \\
\left(\mathrm{~km} \mathrm{~s}^{-1}\right)\end{array}$ & Source $^{a}$ \\
\hline 55340.981 & 0.108 & -38.2 & 0.5 & 0.3 & 77.7 & -0.4 & 0.4 & 16.1 & Fair \\
\hline 55355.937 & 0.131 & -36.4 & -0.5 & 0.3 & 74.0 & -0.2 & 0.4 & 15.2 & Fair \\
\hline 55361.903 & 0.938 & -27.0 & -0.4 & 0.3 & 61.9 & 0.2 & 0.4 & 14.2 & Fair \\
\hline 55367.911 & 0.751 & 30.5 & 0.5 & 0.3 & -14.8 & 0.0 & 0.4 & $\ldots$ & Fair \\
\hline 55367.932 & 0.754 & 28.7 & -0.4 & 0.3 & -13.8 & -0.2 & 0.9 & $\ldots$ & KPNO \\
\hline 55374.803 & 0.683 & 48.2 & 0.2 & 0.3 & -39.1 & 0.1 & 0.4 & 15.6 & Fair \\
\hline 55411.935 & 0.707 & 42.1 & -0.3 & 0.3 & -31.7 & -0.2 & 0.4 & 15.9 & Fair \\
\hline 55459.660 & 0.164 & -30.0 & 0.2 & 0.3 & 66.8 & 0.2 & 0.4 & 16.1 & Fair \\
\hline 55463.701 & 0.710 & 41.1 & -0.4 & 0.3 & -29.7 & 0.6 & 0.9 & 16.4 & KPNO \\
\hline 55469.708 & 0.523 & 61.8 & 0.7 & 0.3 & -57.1 & -0.3 & 0.4 & 14.8 & Fair \\
\hline 55478.624 & 0.729 & 37.6 & 1.3 & 0.3 & -23.2 & 0.2 & 0.4 & $\ldots$ & Fair \\
\hline 55481.749 & 0.152 & -32.5 & -0.1 & 0.3 & 69.4 & -0.2 & 0.4 & 14.1 & Fair \\
\hline 55495.658 & 0.034 & -39.9 & 0.6 & 0.3 & 80.0 & -0.5 & 0.4 & 15.1 & Fair \\
\hline 55505.627 & 0.382 & 34.8 & 0.9 & 0.3 & -19.5 & 0.5 & 0.4 & $\ldots$ & Fair \\
\hline 55516.627 & 0.871 & -7.6 & 0.6 & 0.3 & 36.4 & -0.5 & 0.4 & $\ldots$ & Fair \\
\hline 55626.002 & 0.668 & 51.4 & 0.1 & 0.3 & -42.8 & 0.8 & 0.4 & 15.3 & Fair \\
\hline 55643.961 & 0.098 & -39.5 & 0.1 & 0.3 & 79.5 & 0.2 & 0.4 & 15.4 & Fair \\
\hline 55649.919 & 0.904 & -17.3 & 0.5 & 0.3 & 49.6 & -0.2 & 0.4 & 16.0 & Fair \\
\hline 55664.867 & 0.926 & -23.3 & 0.3 & 0.3 & 57.6 & -0.1 & 0.4 & 14.8 & Fair \\
\hline 55669.979 & 0.617 & 59.5 & 0.2 & 0.3 & -54.2 & 0.1 & 0.4 & 13.4 & Fair \\
\hline 55677.913 & 0.691 & 47.3 & 1.0 & 0.3 & -36.6 & 0.3 & 0.4 & $\ldots$ & Fair \\
\hline 55680.957 & 0.103 & -39.6 & -0.4 & 0.3 & 78.3 & -0.4 & 0.9 & 14.5 & KPNO \\
\hline 55684.832 & 0.627 & 59.1 & 1.0 & 0.3 & -52.2 & 0.6 & 0.4 & 16.7 & Fair \\
\hline 55694.779 & 0.973 & -32.7 & 0.8 & 0.3 & 71.8 & 0.8 & 0.4 & 14.6 & Fair \\
\hline 55698.776 & 0.513 & 59.5 & -0.8 & 0.3 & -55.8 & -0.1 & 0.4 & 14.1 & Fair \\
\hline 55710.816 & 0.142 & -34.2 & -0.1 & 0.3 & 72.1 & 0.2 & 0.4 & 15.4 & Fair \\
\hline 55718.975 & 0.246 & -8.4 & 0.6 & 0.3 & 38.0 & 0.1 & 0.4 & $\ldots$ & Fair \\
\hline 55725.939 & 0.188 & -25.2 & -0.4 & 0.3 & 60.2 & 1.0 & 0.4 & 14.5 & Fair \\
\hline 55731.939 & 1.000 & -38.1 & -0.6 & 0.3 & 76.8 & 0.3 & 0.9 & 15.4 & KPNO \\
\hline 55732.897 & 0.130 & -36.3 & -0.2 & 0.3 & 74.3 & -0.2 & 0.9 & 15.3 & KPNO \\
\hline 55732.924 & 0.133 & -35.1 & 0.4 & 0.3 & 72.9 & -0.9 & 0.4 & 14.9 & Fair \\
\hline 55735.933 & 0.540 & 61.7 & -0.4 & 0.3 & -58.6 & -0.5 & 0.9 & $\ldots$ & KPNO \\
\hline 55743.877 & 0.615 & 59.9 & 0.4 & 0.3 & -54.8 & -0.1 & 0.4 & 14.5 & Fair \\
\hline 55857.686 & 0.012 & -38.8 & 0.1 & 0.3 & 78.8 & 0.5 & 0.4 & 14.4 & Fair \\
\hline 55977.035 & 0.159 & -30.9 & 0.3 & 0.3 & 68.0 & 0.1 & 0.4 & 15.2 & Fair \\
\hline 55997.944 & 0.988 & -35.6 & 0.3 & 0.3 & 74.0 & -0.2 & 0.4 & 14.8 & Fair \\
\hline 56023.876 & 0.496 & 58.8 & 0.5 & 0.3 & -53.4 & -0.3 & 0.4 & 14.8 & Fair \\
\hline 56047.820 & 0.735 & 35.7 & 1.1 & 0.3 & -21.2 & -0.1 & 0.4 & $\ldots$ & Fair \\
\hline 56054.789 & 0.678 & 49.9 & 0.7 & 0.3 & -40.2 & 0.6 & 0.4 & 16.1 & Fair \\
\hline 56060.929 & 0.509 & 59.4 & -0.4 & 0.3 & -55.4 & -0.3 & 0.9 & 16.2 & KPNO \\
\hline 56061.773 & 0.623 & 58.8 & 0.2 & 0.3 & -53.6 & -0.1 & 0.4 & 14.3 & Fair \\
\hline 56061.924 & 0.643 & 55.2 & -0.6 & 0.3 & -49.8 & -0.2 & 0.9 & 14.4 & KPNO \\
\hline 56067.811 & 0.440 & 48.7 & 0.1 & 0.3 & -40.1 & -0.2 & 0.4 & 14.4 & Fair \\
\hline 56076.731 & 0.647 & 55.2 & 0.0 & 0.3 & -48.6 & 0.3 & 0.4 & 15.2 & Fair \\
\hline 56082.769 & 0.463 & 52.7 & -0.6 & 0.3 & -46.0 & 0.3 & 0.4 & 14.2 & Fair \\
\hline 56087.915 & 0.160 & -30.7 & 0.3 & 0.3 & 67.7 & 0.1 & 0.4 & 15.1 & Fair \\
\hline 56095.897 & 0.240 & -10.6 & 0.3 & 0.3 & 40.7 & 0.2 & 0.4 & $\ldots$ & Fair \\
\hline 56100.847 & 0.909 & -19.0 & 0.3 & 0.3 & 51.7 & -0.2 & 0.4 & 15.1 & Fair \\
\hline 56126.855 & 0.428 & 46.5 & 0.7 & 0.3 & -35.7 & 0.5 & 0.4 & 15.2 & Fair \\
\hline 56169.819 & 0.240 & -10.3 & 0.4 & 0.3 & 40.8 & 0.6 & 0.4 & $\ldots$ & Fair \\
\hline 56196.716 & 0.879 & -11.2 & -0.4 & 0.3 & 40.6 & 0.2 & 0.4 & $\ldots$ & Fair \\
\hline 56213.685 & 0.175 & -27.2 & 0.6 & 0.3 & 63.6 & 0.3 & 0.4 & 14.3 & Fair \\
\hline 56228.657 & 0.200 & -21.2 & 0.5 & 0.3 & 55.1 & 0.0 & 0.4 & 15.2 & Fair \\
\hline 56328.034 & 0.645 & 55.8 & 0.3 & 0.3 & -49.1 & 0.1 & 0.4 & 14.4 & Fair \\
\hline 56351.012 & 0.754 & 29.9 & 0.8 & 0.3 & -13.6 & 0.0 & 0.4 & $\ldots$ & Fair \\
\hline 56372.966 & 0.724 & 37.7 & -0.1 & 0.3 & -25.6 & -0.2 & 0.4 & $\ldots$ & Fair \\
\hline 56389.958 & 0.023 & -39.6 & 0.2 & 0.3 & 79.8 & 0.3 & 0.4 & 15.0 & Fair \\
\hline 56400.871 & 0.499 & 59.2 & 0.4 & 0.3 & -53.2 & 0.5 & 0.4 & $\ldots$ & Fair \\
\hline 56414.855 & 0.391 & 37.0 & 0.7 & 0.3 & -23.5 & -0.2 & 0.4 & 15.2 & Fair \\
\hline 56419.828 & 0.064 & -41.0 & 0.1 & 0.3 & 81.0 & -0.3 & 0.4 & 14.9 & Fair \\
\hline 56433.828 & 0.958 & -30.6 & 0.2 & 0.3 & 67.6 & 0.2 & 0.4 & 14.4 & Fair \\
\hline
\end{tabular}

Notes.

${ }^{\mathrm{a}} \mathrm{McD}=\mathrm{McDonald}$ Observatory, Fair $=$ Fairborn Observatory, KPNO $=$ Kitt Peak National Observatory.

${ }^{b}$ Blue wavelength spectrum. 
Table 7

Orbital Elements and Related Parameters of 2 Sge

\begin{tabular}{lcc}
\hline \hline Parameter & Luyten $(1936)$ & This Study \\
\hline$P($ days $)$ & 7.390 & $7.3915807 \pm 0.0000057$ \\
$T(\mathrm{HJD})$ & $\ldots$ & $2454408.85 \pm 0.10$ \\
$T_{0}(\mathrm{JD})$ & $2421047.175 \pm 0.24$ & $\ldots$ \\
$e$ & 0.0 (adopted) & $0.00613 \pm 0.00065$ \\
$\omega(\mathrm{deg})$ & $\ldots$ & $158.4 \pm 5.1$ \\
$K_{1}\left(\mathrm{~km} \mathrm{~s}^{-1}\right)$ & $52.8 \pm 1.0$ & $51.818 \pm 0.052$ \\
$K_{2}\left(\mathrm{~km} \mathrm{~s}^{-1}\right)$ & $77.6 \pm 8.0$ & $70.031 \pm 0.042$ \\
$\gamma\left(\mathrm{km} \mathrm{s}^{-1}\right)$ & $11.2 \pm 0.7$ & $10.950 \pm 0.026$ \\
$m_{1} \sin ^{3} i\left(M_{\odot}\right)$ & $\ldots$ & $0.7963 \pm 0.0011$ \\
$m_{2} \sin ^{3} i\left(M_{\odot}\right)$ & $\ldots$ & $0.5892 \pm 0.0011$ \\
$a_{1} \sin \left(10^{6} \mathrm{~km}\right)$ & $\ldots$ & $5.2668 \pm 0.0053$ \\
$a_{2} \sin i\left(10^{6} \mathrm{~km}\right)$ & $\ldots$ & $7.1179 \pm 0.0042$ \\
Standard error of an unit weight observation $\left(\mathrm{km} \mathrm{s}^{-1}\right)$ & $\ldots$ & 0.28 \\
\hline
\end{tabular}

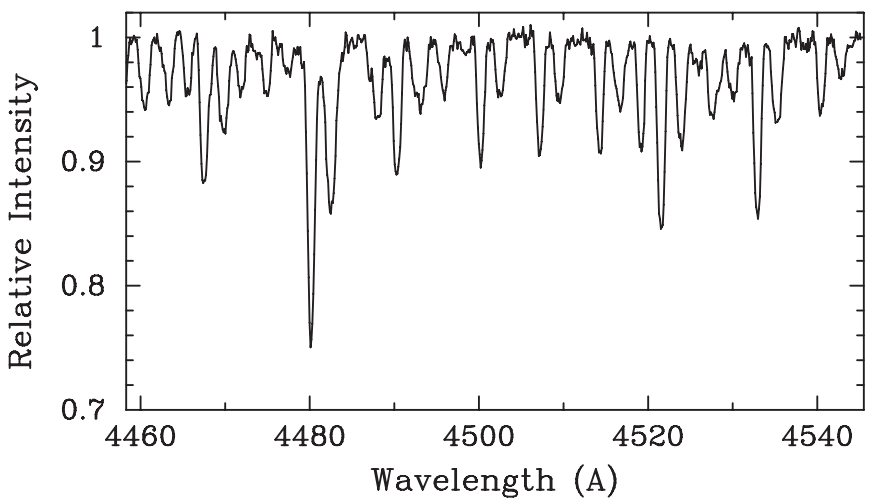

Figure 10. KPNO spectrum of the $4500 \AA$ region of 18 Vul. The strongest feature is the $\mathrm{Mg}$ II line at $4481 \AA$. The more massive primary is the blueshifted component.

\section{4. $18 \mathrm{Vul}$}

For 18 Vul, 62 of our 75 spectra showing double lines were obtained at Fairborn Observatory between 2004 and 2013. Of the remaining spectra, acquired from 2005 through 2008, 11 are from KPNO and two from McDonald Observatory. A KPNO spectrum of $18 \mathrm{Vul}$ in the $4500 \AA$ region is shown in Figure 10. Clearly visible are the moderate but still relatively low rotational broadening and the line strength difference between the two components. All our new radial velocity observations are given in Table 8.

We first obtained four orbital solutions, two for the primary and two for the secondary. Because of the extensive number of Fairborn velocities, those data were analyzed first. Separate orbital solutions result in eccentricities of $0.018 \pm 0.002$ and $0.025 \pm 0.005$ for the primary and secondary, respectively. Following the precepts of Lucy \& Sweeney (1971), we conclude that the orbit is eccentric. However, we note that the longitude of periastron, which for the two solutions should differ by $180^{\circ}$, has a difference of just $43^{\circ}$. Reasons for this apparent discrepancy include the greater difficulty in measuring precise velocities of the broader lined secondary and/or the $\delta$ Scuti pulsation.

Because there are just two observations from McDonald Observatory, we added them to the KPNO velocity set and then determined orbital solutions for the two components with that combined data. The variances of the four solutions resulted in weights of $1.0,0.3$, and 0.3 for the primary velocities from Fairborn, KPNO, and McDonald, respectively. For the secondary, the weights were $0.2,0.05$, and 0.05 for the same

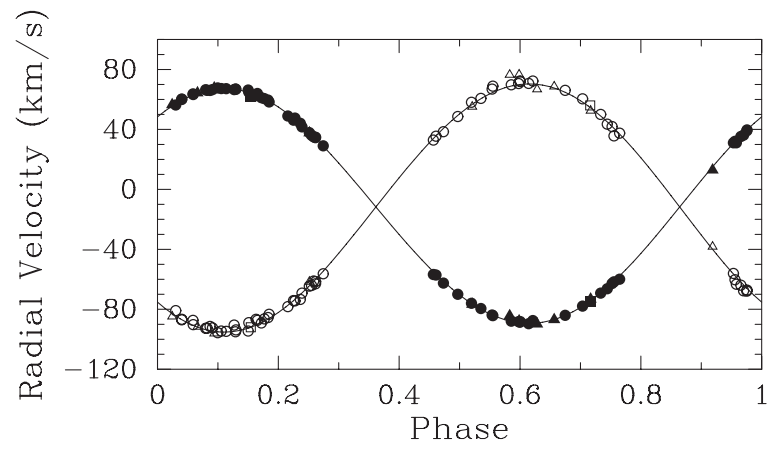

Figure 11. Radial velocities of 18 Vul compared with the computed velocity curves. Filled and open symbols represent the primary and secondary, respectively. Circles $=$ Fairborn Observatory, triangles $=\mathrm{KPNO}$, and squares $=$ McDonald Observatory. Zero phase is a time of periastron passage.

three respective observatories. The center-of-mass velocities of the four data sets agree to within $2.0 \mathrm{~km} \mathrm{~s}^{-1}$ with the $1 \sigma$ uncertainties in the individual values nearly overlapping. The difference between the center-of-mass velocities for the Fairborn primary and secondary velocities is only $0.4 \mathrm{~km} \mathrm{~s}^{-1}$. Thus, we combined all our data into a simultaneous solution for both components, which produced an orbital period of 9.31408 days.

The only other significant set of radial velocities is that of Harper (1918), which were determined from photographic plates obtained at the Dominion Observatory in Ottawa. The standard deviation per plate of the primary velocity is $7.2 \mathrm{~km} \mathrm{~s}^{-1}$, resulting in such low weights that they do not significantly improve the value of the period. Thus, in Table 9 we present the orbital elements from our velocities only. Our primary and secondary velocities are compared with the computed velocity curves in Figure 11. In that plot, zero phase is a time of periastron passage. In Figure 12 the velocity residuals of the two components are plotted versus phase. Compared with the results of our other three systems, the residuals for both components of $18 \mathrm{Vul}$ are much larger. This is primarily due to the substantially greater line broadening of the two components, but may also result from pulsation in one or both components.

Harper (1918) did not provide uncertainties for his derived orbital elements, and so in Table 9 we compare our results to the reanalysis of Harper's velocities that was computed by Luyten (1936). The uncertainty of our period and semi-amplitudes is significantly improved. Our semi-amplitudes are both smaller than those of Harper's with that for the secondary being reduced 
Table 8

Radial Velocities of $18 \mathrm{Vul}$

\begin{tabular}{|c|c|c|c|c|c|c|c|c|}
\hline $\begin{array}{l}\text { Hel. Julian Date } \\
\text { (HJD - 2,400,000) }\end{array}$ & Phase & $\begin{array}{c}V_{1} \\
\left(\mathrm{~km} \mathrm{~s}^{-1}\right)\end{array}$ & $\begin{array}{l}(O-C)_{1} \\
\left(\mathrm{~km} \mathrm{~s}^{-1}\right)\end{array}$ & $\mathrm{Wt}_{1}$ & $\begin{array}{c}V_{2} \\
\left(\mathrm{~km} \mathrm{~s}^{-1}\right)\end{array}$ & $\begin{array}{c}(O-C)_{2} \\
\left(\mathrm{~km} \mathrm{~s}^{-1}\right)\end{array}$ & $\mathrm{Wt}_{2}$ & Source $^{\mathrm{a}}$ \\
\hline 53311.719 & 0.150 & 66.3 & 1.5 & 1.0 & -94.5 & -1.9 & 0.20 & Fair \\
\hline 53312.695 & 0.255 & 36.1 & -0.6 & 1.0 & -64.1 & -1.2 & 0.20 & Fair \\
\hline 53354.614 & 0.755 & -61.7 & -0.3 & 1.0 & 35.7 & -5.1 & 0.20 & Fair \\
\hline $53638.655^{\mathrm{b}}$ & 0.251 & 37.7 & -0.4 & 0.3 & -61.6 & 2.7 & 0.05 & KPNO \\
\hline 53689.554 & 0.716 & -75.0 & -0.5 & 0.3 & 56.0 & 1.3 & 0.05 & $\mathrm{McD}$ \\
\hline 53855.967 & 0.583 & -84.3 & 3.5 & 0.3 & 76.5 & 7.8 & 0.05 & KPNO \\
\hline $54005.678^{b}$ & 0.657 & -86.9 & -0.1 & 0.3 & 68.7 & 1.0 & 0.05 & KPNO \\
\hline 54047.571 & 0.154 & 62.0 & -2.3 & 0.3 & -92.0 & 0.0 & 0.05 & $\mathrm{McD}$ \\
\hline 54223.976 & 0.094 & 67.7 & 0.8 & 0.3 & -96.1 & -1.3 & 0.05 & KPNO \\
\hline 54265.931 & 0.598 & -87.7 & 1.2 & 0.3 & 76.6 & 6.7 & 0.05 & KPNO \\
\hline 54269.897 & 0.024 & 56.5 & 0.9 & 0.3 & -84.8 & -1.9 & 0.05 & KPNO \\
\hline 54367.663 & 0.521 & -76.7 & -0.2 & 0.3 & 55.2 & -1.6 & 0.05 & KPNO \\
\hline $54368.665^{\mathrm{b}}$ & 0.628 & -89.8 & -0.7 & 0.3 & 66.8 & -3.3 & 0.05 & KPNO \\
\hline 54408.625 & 0.919 & 12.9 & -2.2 & 0.3 & -38.2 & 1.8 & 0.05 & KPNO \\
\hline $54586.967^{\mathrm{b}}$ & 0.066 & 64.4 & 0.2 & 0.3 & -91.6 & 0.3 & 0.05 & KPNO \\
\hline 54732.738 & 0.717 & -73.2 & 1.1 & 0.3 & 52.5 & -1.9 & 0.05 & KPNO \\
\hline 55275.966 & 0.040 & 60.3 & 0.8 & 1.0 & -86.7 & 0.3 & 0.20 & Fair \\
\hline 55293.899 & 0.966 & 35.3 & -0.5 & 1.0 & -63.9 & -2.0 & 0.20 & Fair \\
\hline 55314.857 & 0.216 & 49.0 & -1.4 & 1.0 & -78.2 & -0.8 & 0.20 & Fair \\
\hline 55331.848 & 0.040 & 60.0 & 0.6 & 1.0 & -87.0 & -0.1 & 0.20 & Fair \\
\hline 55341.772 & 0.105 & 67.3 & 0.0 & 1.0 & -94.5 & 0.7 & 0.20 & Fair \\
\hline 55377.817 & 0.975 & 39.1 & -0.5 & 1.0 & -67.9 & -1.9 & 0.20 & Fair \\
\hline 55401.833 & 0.554 & -83.8 & 0.0 & 1.0 & 66.9 & 2.4 & 0.20 & Fair \\
\hline 55412.820 & 0.733 & -69.2 & -0.1 & 1.0 & 50.0 & 1.0 & 0.20 & Fair \\
\hline 55459.683 & 0.765 & -59.9 & -2.2 & 1.0 & 37.5 & 0.6 & 0.20 & Fair \\
\hline 55513.613 & 0.555 & -84.2 & -0.2 & 1.0 & 68.9 & 4.2 & 0.20 & Fair \\
\hline 55554.602 & 0.956 & 32.1 & 0.4 & 1.0 & -60.3 & -2.7 & 0.20 & Fair \\
\hline 55631.962 & 0.262 & 34.5 & 0.4 & 1.0 & -63.0 & -2.9 & 0.20 & Fair \\
\hline 55640.959 & 0.227 & 47.6 & 0.9 & 1.0 & -74.4 & -1.0 & 0.20 & Fair \\
\hline 55648.919 & 0.082 & 66.1 & 0.1 & 1.0 & -92.6 & 1.3 & 0.20 & Fair \\
\hline 55653.941 & 0.621 & -87.5 & 1.8 & 1.0 & 72.3 & 2.0 & 0.20 & Fair \\
\hline 55662.923 & 0.586 & -87.9 & 0.1 & 1.0 & 69.8 & 0.8 & 0.20 & Fair \\
\hline 55667.985 & 0.129 & 67.0 & 0.2 & 1.0 & -95.0 & -0.4 & 0.20 & Fair \\
\hline 55675.871 & 0.976 & 39.7 & -0.1 & 1.0 & -67.2 & -1.1 & 0.20 & Fair \\
\hline 55680.936 & 0.520 & -76.0 & 0.1 & 1.0 & 58.1 & 1.7 & 0.20 & Fair \\
\hline 55704.782 & 0.080 & 66.5 & 0.7 & 1.0 & -92.7 & 0.9 & 0.20 & Fair \\
\hline 55732.910 & 0.100 & 67.7 & 0.6 & 1.0 & -95.6 & -0.6 & 0.20 & Fair \\
\hline 55844.809 & 0.114 & 67.2 & -0.1 & 1.0 & -94.7 & 0.5 & 0.20 & Fair \\
\hline 55892.660 & 0.251 & 37.9 & -0.3 & 1.0 & -64.5 & -0.1 & 0.20 & Fair \\
\hline 55967.034 & 0.236 & 44.1 & 0.5 & 1.0 & -73.4 & -3.2 & 0.20 & Fair \\
\hline 55989.028 & 0.598 & -88.2 & 0.7 & 1.0 & 70.6 & 0.7 & 0.20 & Fair \\
\hline 56011.950 & 0.059 & 63.7 & 0.7 & 1.0 & -87.4 & 3.3 & 0.20 & Fair \\
\hline 56012.922 & 0.163 & 62.5 & -0.5 & 1.0 & -86.7 & 3.9 & 0.20 & Fair \\
\hline 56040.885 & 0.165 & 64.0 & 1.4 & 1.0 & -87.4 & 2.8 & 0.20 & Fair \\
\hline 56058.821 & 0.091 & 66.6 & -0.1 & 1.0 & -92.4 & 2.2 & 0.20 & Fair \\
\hline 56064.907 & 0.744 & -66.3 & -0.9 & 1.0 & 43.5 & -1.6 & 0.20 & Fair \\
\hline 56068.889 & 0.172 & 61.2 & -0.2 & 1.0 & -89.1 & -0.1 & 0.20 & Fair \\
\hline 56077.784 & 0.127 & 66.6 & -0.3 & 1.0 & -90.5 & 4.3 & 0.20 & Fair \\
\hline 56086.727 & 0.087 & 66.0 & -0.4 & 1.0 & -91.4 & 2.9 & 0.20 & Fair \\
\hline 56097.785 & 0.274 & 29.1 & 0.3 & 1.0 & -56.3 & -1.7 & 0.20 & Fair \\
\hline 56100.816 & 0.600 & -88.6 & 0.4 & 1.0 & 72.3 & 2.3 & 0.20 & Fair \\
\hline 56101.783 & 0.704 & -77.9 & 0.0 & 1.0 & 60.4 & 2.1 & 0.20 & Fair \\
\hline 56227.724 & 0.225 & 46.2 & -1.3 & 1.0 & -74.2 & 0.0 & 0.20 & Fair \\
\hline 56236.646 & 0.183 & 59.6 & 0.5 & 1.0 & -85.5 & 1.1 & 0.20 & Fair \\
\hline 56274.635 & 0.262 & 34.8 & 0.8 & 1.0 & -61.5 & -1.4 & 0.20 & Fair \\
\hline 56342.025 & 0.497 & -70.0 & -0.5 & 1.0 & 48.6 & -0.8 & 0.20 & Fair \\
\hline 56352.994 & 0.675 & -84.0 & 0.1 & 1.0 & 66.1 & 1.3 & 0.20 & Fair \\
\hline 56374.933 & 0.030 & 56.3 & -0.8 & 1.0 & -80.9 & 3.6 & 0.20 & Fair \\
\hline 56392.890 & 0.958 & 31.1 & -1.6 & 1.0 & -63.3 & -4.7 & 0.20 & Fair \\
\hline 56406.888 & 0.461 & -57.1 & -0.5 & 1.0 & 35.5 & -0.3 & 0.20 & Fair \\
\hline 56416.895 & 0.535 & -79.5 & 0.5 & 1.0 & 60.7 & 0.2 & 0.20 & Fair \\
\hline 56418.917 & 0.752 & -62.8 & -0.3 & 1.0 & 41.9 & -0.1 & 0.20 & Fair \\
\hline 56420.787 & 0.953 & 31.1 & 0.5 & 1.0 & -56.1 & 0.4 & 0.20 & Fair \\
\hline 56420.944 & 0.970 & 36.2 & -1.4 & 1.0 & -67.7 & -3.9 & 0.20 & Fair \\
\hline
\end{tabular}


Table 8

(Continued)

\begin{tabular}{lcccccccc}
\hline \hline $\begin{array}{l}\text { Hel. Julian Date } \\
(\mathrm{HJD}-2,400,000)\end{array}$ & Phase & $\begin{array}{c}V_{1} \\
\left(\mathrm{~km} \mathrm{~s}^{-1}\right)\end{array}$ & $\begin{array}{c}(O-C)_{1} \\
\left(\mathrm{~km} \mathrm{~s}^{-1}\right)\end{array}$ & $\mathrm{Wt}_{1}$ & $\begin{array}{c}V_{2} \\
\left(\mathrm{~km} \mathrm{~s}^{-1}\right)\end{array}$ & $\begin{array}{r}(O-C)_{2} \\
\left(\mathrm{~km} \mathrm{~s}^{-1}\right)\end{array}$ & $\mathrm{Wt}_{2}$ & Source $^{\mathrm{a}}$ \\
\hline 56421.773 & 0.059 & 63.3 & 0.2 & 1.0 & -90.2 & 0.6 & 0.20 & Fair \\
56422.869 & 0.177 & 60.6 & 0.2 & 1.0 & -86.4 & 1.6 & 0.20 & Fair \\
56422.944 & 0.185 & 58.3 & -0.4 & 1.0 & -83.3 & 2.8 & 0.20 & Fair \\
56426.816 & 0.601 & -88.2 & 0.8 & 1.0 & 72.0 & 2.0 & 0.20 & Fair \\
56426.944 & 0.614 & -89.6 & -0.3 & 1.0 & 70.7 & 0.3 & 0.20 & Fair \\
56431.747 & 0.130 & 66.8 & 0.1 & 1.0 & -93.7 & 0.9 & 0.20 & Fair \\
56431.945 & 0.151 & 64.8 & 0.1 & 1.0 & -88.9 & 3.6 & 0.20 & Fair \\
56432.765 & 0.239 & 41.7 & -0.9 & 1.0 & -69.2 & -0.1 & 0.20 & Fair \\
56432.945 & 0.259 & 35.0 & -0.3 & 1.0 & -61.1 & 0.3 & 0.20 & Fair \\
56434.790 & 0.457 & -56.8 & -1.9 & 1.0 & 32.9 & -1.1 & 0.20 & Fair \\
56434.945 & 0.473 & -62.5 & -1.2 & 1.0 & 38.5 & -2.2 & 0.20 & Fair \\
\hline
\end{tabular}

Notes.

${ }^{\mathrm{a}}$ Fair $=$ Fairborn Observatory, $\mathrm{KPNO}=$ Kitt Peak National Observatory, and McD $=$ McDonald Observatory.

${ }^{\mathrm{b}}$ Blue wavelength spectrum.

Table 9

Orbital Elements and Related Parameters of 18 Vul

\begin{tabular}{lcc}
\hline \hline Parameter & Luyten $(1936)$ & This Study \\
\hline$P$ (days) & 9.316 & $9.314077 \pm 0.000039$ \\
$T$ (HJD) & $\ldots$ & $2454875.09 \pm 0.25$ \\
$T_{0}(\mathrm{JD})$ & $2421103.17 \pm 0.03$ & $\ldots$ \\
$e$ & 0.0 (adopted) & $0.0116 \pm 0.0019$ \\
$\omega(\mathrm{deg})$ & $\ldots$ & $319.4 \pm 0.97$ \\
$K_{1}\left(\mathrm{~km} \mathrm{~s}^{-1}\right)$ & $78.5 \pm 1.4$ & $78.33 \pm 0.13$ \\
$K_{2}\left(\mathrm{~km} \mathrm{~s}^{-1}\right)$ & $86.3 \pm 1.4$ & $82.80 \pm 0.29$ \\
$\gamma\left(\mathrm{km} \mathrm{s}^{-1}\right)$ & $-13.0 \pm 1.0$ & $-11.70 \pm 0.11$ \\
$m_{1} \sin ^{3} i\left(M_{\odot}\right)$ & $\ldots$ & $2.074 \pm 0.014$ \\
$m_{2} \sin 3\left(M_{\odot}\right)$ & $\ldots$ & $1.9622 \pm 0.0090$ \\
$a_{1} \sin i\left(10^{6} \mathrm{~km}\right)$ & $\ldots$ & $10.032 \pm 0.017$ \\
$a_{2} \sin i\left(10^{6} \mathrm{~km}\right)$ & $\ldots$ & $10.604 \pm 0.037$ \\
Standard error of an unit weight observation $\left(\mathrm{km} \mathrm{s}^{-1}\right)$ & $\ldots$ & 0.86 \\
\hline
\end{tabular}

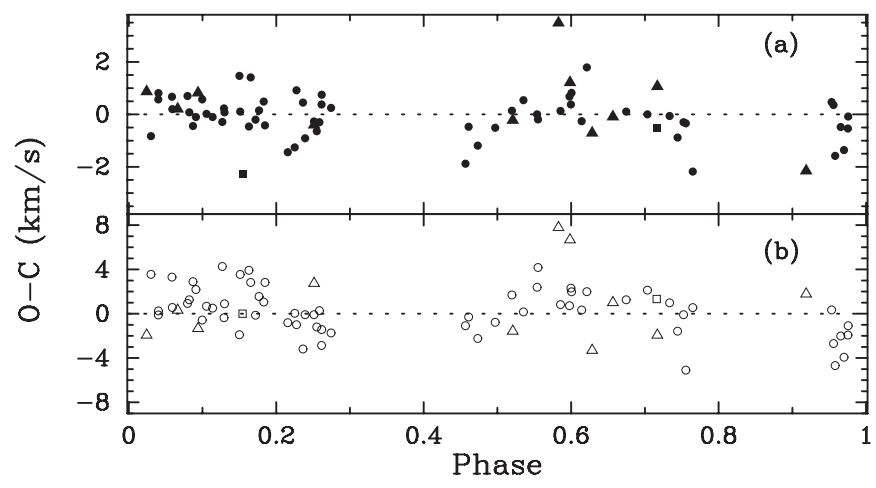

Figure 12. 18 Vul radial velocity residuals vs. orbital phase. Panel (a) shows residuals of the primary, while panel (b) is of the secondary. Circles $=$ Fairborn Observatory, triangles $=\mathrm{KPNO}$, and squares $=$ McDonald Observatory. Zero phase is a time of periastron passage.

by $4 \%$. While this results in smaller minimum masses, both stars still have minimum values that are about $2 M_{\odot}$.

\section{SPECTRAL TYPES AND MAGNITUDE DIFFERENCE}

Strassmeier \& Fekel (1990) identified several luminositysensitive and temperature-sensitive line ratios in the 6430-6465 A region. They employed those critical line ratios and the general appearance of the spectrum as spectral-type criteria. For our A-star binaries we have examined the gen- eral appearance of our KPNO spectra in both the $4500 \AA$ and $6430 \AA$ regions to determine spectral classes. However, the obvious abundance peculiarities in many of the components of our A-star systems make normal spectral classification very difficult in our two limited $84 \AA$ regions. Also, in those two regions the line ratios of $\mathrm{A}$ and $\mathrm{F}$ spectral class stars have little sensitivity to luminosity. Thus, it is necessary to determine the luminosity class by computing the absolute visual magnitude with the Hipparcos parallax. Those magnitudes, converted to luminosities, and the adopted temperatures of the stars are then compared to evolutionary tracks. Because such luminosity classes are not assigned from the appearance of the spectra, we have used words such as dwarf or subgiant to describe the luminosity class rather than using Roman numerals.

The comparison spectra for our binaries are mostly slowly rotating A stars from the list of Abt \& Morrell (1995) plus a few $\mathrm{F}$ and $\mathrm{G}$ stars from Fekel (1997). Those spectra were acquired at KPNO with the same telescope, spectrograph, and detector as our binary star spectra. In an attempt to reproduce the binary spectra, various comparison-star combinations were rotationally broadened when necessary, shifted in radial velocity to mimic the separation of the two components, appropriately weighted, and added together with a computer program developed by Huenemoerder \& Barden (1984) and Barden (1985).

From the equivalent widths of line pairs, we determined the continuum intensity ratio of the binary components at $4500 \AA$ in our KPNO spectra. The continuum intensity ratio at $5700 \AA$ is 
from our Fairborn Observatory spectra that were measured with the A-star line list, while that same ratio at $6000 \AA$ is from our solar-type star line list. The continuum intensity ratio is equal to the luminosity ratio and converted to a magnitude difference if the stars have the same or very similar spectral types. If, on the other hand, the components are main-sequence stars of very different spectral types, then the lines of the secondary are intrinsically significantly stronger than those of the primary. In that case, the continuum intensity ratio becomes a minimum magnitude difference. Abundance peculiarities, such as those in Am stars, make any such magnitude difference even more uncertain.

Abt \& Morrell (1995) stated that based on their hydrogen lines, classical Am stars have spectral classes ranging from A4 to F1. The Am stars are identified as peculiar because relative to the hydrogen classification, various elements including $\mathrm{Fe}$ and $\mathrm{Sr}$ are stronger than expected, leading to a later spectral class, and other elements like $\mathrm{Ca}$ and $\mathrm{Sc}$ have weaker lines than expected, resulting in an earlier spectral class (Abt \& Morrell 1995). Our limited regions around $4500 \AA$ and $6430 \AA$ contain no hydrogen lines. In addition, the overabundance and underabundance peculiarities vary from star to star. Thus, the spectral class analysis of our binary systems has met with limited success especially for those components that are Am stars.

\subsection{HR 1528}

As noted by Bidelman (Abt \& Bidelman 1969), HR 1528 has pronounced Am star characteristics. Abt \& Morrell (1995) classified the combined spectrum of HR 1528 as an Am star with A7, F0, and F2 spectral classes for its Ca K, hydrogen, and metal lines, respectively. Thus, because HR 1528 is an Am star, our analysis of its spectrum is limited. Comparison with reference stars indicates that the $\mathrm{Fe}$ I lines at red wavelengths are similar in strength to those of HR 5075 (F2 V; Abt \& Morrell $1995)$, while the $B-V$ color indicates a spectral type of $A 8 / 9 \mathrm{~V}$.

From our Fairborn observations, the secondary to primary line strength ratio at $\sim 6000 \AA$ is 2.37 . However, the Am star nature of the primary with its strengthened Fe lines makes this value suspect. In addition, such a large magnitude difference between the primary and the secondary means that the lines of the secondary are very weak. Thus, the secondary spectral class is not well determined. From the secondary to primary mass ratio of 0.66 and the canonical properties tables of Gray (1992), we estimate a $V$ magnitude difference of $2.1 \pm 0.4$ (Gray 1992) suggesting that the spectral type of the secondary is a late-F or early-G dwarf. The evolutionary track comparison discussed in Section 6.1 indicates that both components are main-sequence stars.

\subsection{HR 6993}

Although its sharp lines would suggest that HR 6993 is an early-type Am star, Cowley et al. (1969), Floquet (1975), and Levato \& Abt (1978) classified the combined spectrum as a normal A2 V star. Based on a $13 \mathrm{~A} \mathrm{~mm}^{-1}$ spectrogram that resolved the narrow lines of the binary, Geary \& Abt (1970) concluded that while the primary has a normal spectrum, the secondary appears to be an Am star.

Both our blue and red wavelength KPNO spectra show that most of the line pairs are similar in strength. HR 4378 (A2 Vs; Abt \& Morrell 1995) provides a reasonably good fit to the spectrum of HR 6993 at both blue and red wavelengths, but the $\mathrm{Ca}$ I lines of the secondary are indeed weaker than expected from the general fit.
At $5700 \AA$ the continuum intensity ratio of the secondary to the primary is 0.705 , which results in a continuum magnitude difference of $0.4 \pm 0.1$, while at $4500 \AA$ the intensity ratio is 0.764 , resulting in a continuum magnitude difference of $0.3 \pm$ 0.1 . This value is similar to that of Petrie (1950), who analyzed a blue photographic plate and found a difference of $0.16 \pm 0.06$ at a mean wavelength of $4450 \AA$ from just three line pairs.

The secondary has a very similar spectral class to the primary, and so we adopt a $V$ magnitude difference of $0.4 \pm 0.1$. As discussed in Section 6.2, visual magnitudes from the reanalyzed Hipparcos parallax (van Leeuwen 2007) indicate that both components are on the main sequence.

$$
\text { 5.3. } 2 \text { Sge }
$$

The difficulty of identifying peculiarities in early-type A stars from low-dispersion classification spectra is exemplified by 2 Sge. Cowley et al. (1969) indicated that 2 Sge is a mild metallic-lined A star, and Bidelman (Abt \& Bidelman 1969) concluded that it is a probable Am star, while Bertaud (1970) classified it as a full-fledged Am star with its $\mathrm{Ca} \mathrm{K}$ line being A2 and its metal lines A7. On the other hand, other classifiers have found no spectrum peculiarities. Levato (1975) assigned a spectral type of A2 III-IV, while Abt \& Morrell (1995) classified its spectral type as A1 III.

Stickland (1973) performed a spectroscopic analysis that indicated an overabundance of $\mathrm{Fe}$ and an underabundance of $\mathrm{Sc}$ in both components as expected for Am stars. Our examination of the $6430 \AA$ region confirms that both shortperiod components have an overabundance of $\mathrm{Fe}$ while $\mathrm{Ca}$ appears to be underabundant. Very weak lines of the close visual secondary, discovered by the Hipparcos team (Perryman et al. 1997), are also visible in this wavelength region (Figure 6).

At $4500 \AA$ the continuum intensity ratio is 0.336 , which converts to a magnitude difference of $1.2 \pm 0.1$. For lines centered at $5700 \AA$, an intensity ratio of 0.479 produces a $0.8 \pm$ 0.1 mag difference. These values assume that the Fe surface abundances of the two stars are the same, which, because both components are Am stars, is not necessarily the case. The combined $B-V$ value and the mass ratio compared with canonical values in Table B1 of Gray (1992) suggest spectral classes of A2 and A7 and a $V$ magnitude difference of $1.0 \pm$ 0.2, which we adopt. From our discussion in Section 6.3, both components appear to be main-sequence stars.

\section{4. $18 \mathrm{Vul}$}

Spectral types of 18 Vul include A1 IV (Svolopoulos 1961), A2 IV (Abt \& Morrell 1995), A3 V (Cowley et al. 1969), and A3 III (Osawa 1959). Bidelman (Abt \& Bidelman 1969) concluded that its spectrum was that of a normal A star with no peculiarities.

Our blue and red wavelength spectra show that the components are somewhat similar in strength with the weaker lined secondary rotating more rapidly. Thus, we compared its spectrum with the reference stars HR 4378 (A2 Vs; Abt \& Morrell 1995) and 68 Tau (A2 IV; Abt \& Morrell 1995). An abundance analysis by Pintado \& Adelman (2003) has shown that 68 Tau is an early-type Am star with stronger Fe lines and weaker $\mathrm{Ca}$ lines than expected for its hydrogen spectral type. In general, the Fe lines of 18 Vul are fitted better by 68 Tau than by HR 4378 . However, the Ca lines of $18 \mathrm{Vul}$ are similar in strength to HR 4378 and significantly stronger than those of 68 Tau. Thus, we find no obvious abundance peculiarities.

At $5700 \AA$ the continuum intensity ratio of the secondary to the primary is 0.601 , which results in a continuum magnitude 
Table 10

Derived Properties of the Binary Components

\begin{tabular}{lcccc}
\hline \hline Parameter & HR 1528 & HR 6993 & 2 Sge & 18 Vul \\
\hline$V_{1}(\mathrm{mag})$ & $5.99 \pm 0.05$ & $6.33 \pm 0.10$ & $6.63 \pm 0.20$ & $6.00 \pm 0.10$ \\
$V_{2}(\mathrm{mag})$ & $8.09 \pm 0.40$ & $6.73 \pm 0.10$ & $7.63 \pm 0.20$ & $6.60 \pm 0.10$ \\
$L_{1}\left(L_{\odot}\right)$ & $21.3 \pm 2.0$ & $60.5 \pm 8.5$ & $22.6 \pm 4.9$ & $51.6 \pm 6.0$ \\
$L_{2}\left(L_{\odot}\right)$ & $3.3 \pm 1.2$ & $41.9 \pm 5.9$ & $8.4 \pm 1.8$ & $31.1 \pm 3.6$ \\
$R_{1}\left(R_{\odot}\right)$ & $2.7 \pm 0.2$ & $3.4 \pm 0.3$ & $2.0 \pm 0.3$ & $3.5 \pm 0.3$ \\
$R_{2}\left(R_{\odot}\right)$ & $1.7 \pm 0.3$ & $2.8 \pm 0.2$ & $1.6 \pm 0.2$ & $2.4 \pm 0.2$ \\
$M_{1}{ }^{\mathrm{a}}\left(M_{\odot}\right)$ & 1.95 & 2.5 & 2.08 & 2.4 \\
$M_{2}{ }^{\mathrm{a}}\left(M_{\odot}\right)$ & $1.2: \mathrm{b}$ & 2.35 & 1.67 & 2.2 \\
\hline
\end{tabular}

Notes.

${ }^{a}$ Masses are estimated from evolutionary tracks in Figure 13.

b The colon indicates a very uncertain value.

difference of $0.55 \pm 0.1$, while at $4500 \AA$ the intensity ratio is 0.535 , resulting in a continuum magnitude difference of $0.7 \pm$ 0.1 . The spectral classes for the two stars appear to be similar, and so we adopt a $V$ magnitude difference of $0.6 \pm 0.1$. From our discussion in Section 6.4, we classify the primary and secondary as dwarf stars.

\section{BASIC PROPERTIES}

\subsection{HR 1528}

To determine the basic properties of HR 1528, we use the Stefan-Boltzmann law. We begin by adopting a $V$ mag of 5.84 and a $B-V$ color of 0.250 mag from the Hipparcos catalog (Perryman et al. 1997). With our adopted $V$ magnitude difference of $2.1 \pm 0.4$, the individual $V$ magnitudes are 5.99 and 8.09 for the primary and secondary, respectively. The new Hipparcos parallax reduction by van Leeuwen (2007) produces a value of $11.94 \pm 0.49$ mas and corresponds to a distance of $83.8 \pm 3.5 \mathrm{pc}$. At such a distance we assume that the interstellar extinction is negligible. The resulting absolute magnitudes are $M_{V}=1.38 \pm$ $0.10 \mathrm{mag}$ and $M_{V}=3.48 \pm 0.40 \mathrm{mag}$ for the primary and secondary, respectively. The uncertainty in the luminosity ratio has a much greater effect on the secondary than the primary. Given the large magnitude difference, the $B-V$ color of the primary will be only slightly smaller than the combined value, and we adopt a value of 0.23 for it. The minimum mass of the secondary indicates that it may well be a late-F or early-G star, a result consistent with the adopted magnitude difference, and so we use 0.56 for its $B-V$. For the two components, the effective temperatures and bolometric corrections come from Table 3 of Flower (1996). From spectral type and temperature calibration uncertainties, we estimate effective temperature uncertainties of $\pm 200 \mathrm{~K}$ for both components. Then our luminosities of the primary and secondary are $L_{1}=21.3 \pm 2.0 L_{\odot}$ and $L_{2}=3.3 \pm$ $1.2 L_{\odot}$, respectively, while the radii are $R_{1}=2.7 \pm 0.2 R_{\odot}$ and $R_{2}=1.7 \pm 0.3 R_{\odot}$, respectively. These derived basic parameters are listed in Table 10.

Our estimated effective temperatures and luminosities for the components of HR 1528 are compared with the solarabundance evolutionary tracks of Girardi et al. (2000) in Figure 13. Although the surface enhancement of Fe and other metals may make Am stars such as the primary of HR 1528 appear to be metal rich, that component is still on the main sequence. When compared to those of solar composition, metalrich tracks for the same masses (Girardi et al. 2000) are shifted to cooler temperatures and lower luminosities. This results in a position for the Am star component that is closer to the zeroage main sequence but of higher mass. The much lower mass

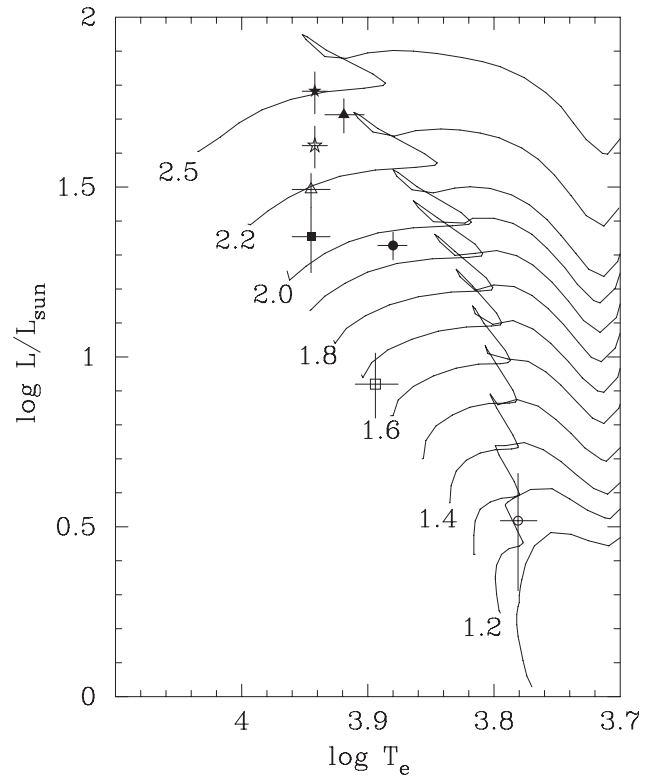

Figure 13. Positions of the components of HR 1528 (circles), HR 6993 (stars), 2 Sge (squares), and $18 \mathrm{Vul}$ (triangles) compared with the $1.1-2.5 M_{\odot}$ solarabundance evolutionary tracks of Girardi et al. (2000). The more massive star in each system corresponds to the filled symbol. Our estimated uncertainties are shown.

secondary is also a main-sequence star. Masses estimated from the evolutionary tracks are given in Table 10 .

\subsection{HR 6993}

As we did for HR 1528, we estimate the basic properties of HR 6993 using the Stefan-Boltzmann law. We first adopt a $V$ mag of 5.76 and a $B-V$ color of 0.067 mag from the Hipparcos catalog (Perryman et al. 1997). With a $\Delta V$ of $0.4 \pm$ $0.1 \mathrm{mag}$, the $V$ magnitudes of the primary and secondary are 6.33 and $6.73 \mathrm{mag}$, respectively. The system parallax of $6.23 \pm 0.033$ mas (van Leeuwen 2007) results in a distance of $160.5 \pm 8.6 \mathrm{pc}$. Although at this distance there might be a modest amount of reddening, the spectral type is consistent with the $B-V$ color and $\mathrm{H} \beta$ value (Stokes 1972; Crawford 1979), and so we assume that there is no reddening for this star system. The components' absolute magnitudes are $M_{V}=$ $0.30 \pm 0.15$ and $M_{V}=0.70 \pm 0.15$ for the primary and secondary, respectively. Table 3 of Flower (1996) then provides the bolometric corrections and effective temperatures that we adopt for the stars. The effective temperature uncertainties are estimated to be $\pm 200 \mathrm{~K}$ for both components. We then determine luminosities of $L_{1}=60.5 \pm 8.5 L_{\odot}$ and $L_{2}=41.9 \pm 5.9 L_{\odot}$ and radii of $R_{1}=3.4 \pm 0.3 R_{\odot}$ and $R_{2}=2.8 \pm 0.2 R_{\odot}$ for the primary and secondary, respectively. These derived basic parameters are given in Table 10.

Our estimated temperatures and luminosities for the two stars are plotted in a Hertzsprung-Russell (H-R) diagram (Figure 13) and compared to the solar-abundance evolutionary tracks of Girardi et al. (2000). The positions of the two components in the H-R diagram indicate that both stars are dwarfs. Masses estimated from the evolutionary tracks are listed in Table 10.

$$
\text { 6.3. } 2 \text { Sge }
$$

From the Hipparcos catalog (Perryman et al. 1997), the $V$ magnitude and $B-V$ color of the 2 Sge system are 6.27 and 0.075 , respectively. With the $1.0 \pm 0.2$ magnitude difference adopted in Section 5.3, the apparent $V$ magnitudes are 6.63 and 
7.63 for the short-period primary and secondary, respectively. The Hipparcos parallax reduction by van Leeuwen (2007) results in a value of $8.92 \pm 0.52$ mas and corresponds to a distance of $112.1 \pm 6.6 \mathrm{pc}$. At such a distance we assume that the interstellar extinction is negligible. The apparent magnitudes and parallax then result in absolute magnitudes $M_{V}$ of $1.38 \pm 0.24$ and $2.38 \pm 0.24$ for the primary and secondary, respectively. From Johnson (1966) we assume $B-V$ colors of 0.06 and 0.19 for the two components, respectively. Table 3 of Flower (1996) then provides the bolometric corrections and effective temperatures that we adopt for the stars. The effective temperature uncertainties are estimated to be $\pm 300 \mathrm{~K}$ for both components. The resulting luminosities are $L_{1}=22.6 \pm 4.9 L_{\odot}$ and $L_{2}=$ $8.4 \pm 1.8 L_{\odot}$, and the radii are $R_{1}=2.0 \pm 0.3 R_{\odot}$ and $R_{2}=$ $1.6 \pm 0.2 R_{\odot}$ for the primary and secondary, respectively. These derived basic properties are summarized in Table 10 .

In Figure 13 we plot our estimated temperatures and luminosities for the short-period components of 2 Sge where those points are compared to the solar-abundance evolutionary tracks of Girardi et al. (2000). Once again, both stars appear to be dwarfs. Masses estimated from the evolutionary tracks are given in Table 10.

\section{4. $18 \mathrm{Vul}$}

For the $18 \mathrm{Vul}$ system, we adopt a $V$ magnitude and $B-V$ color of 5.51 and 0.087, respectively (Perryman et al. 1997). Using our $V$ magnitude difference of $0.6 \pm 0.1$, we compute individual apparent $V$ magnitudes of 6.00 and 6.60. The new reduction of the Hipparcos parallax produces a value of $7.70 \pm$ 0.27 mas (van Leeuwen 2007), which corresponds to a distance of $129.9 \pm 4.6 \mathrm{pc}$. At such a distance, there may be a slight amount of interstellar extinction. However, the $B-V$ color and spectral type are consistent, so we have not included any reddening. We then combine the apparent magnitudes and the parallax to obtain absolute magnitudes $M_{V}=0.43 \pm$ 0.13 and $M_{V}=1.03 \pm 0.13$ for the primary and secondary, respectively. The difference in spectral class between the two components is not large, perhaps a couple subclasses at most. Thus, from Johnson (1966) we assume $B-V$ colors of 0.12 and 0.06 for the primary and secondary, respectively. We then use Table 3 of Flower (1996) to obtain the bolometric corrections and effective temperatures of the two components, where the effective temperature uncertainty is estimated to be $\pm 300 \mathrm{~K}$. The resulting luminosities of the primary and secondary are $L_{1}=51.6 \pm 6.0 L_{\odot}$ and $L_{2}=31.1 \pm 3.6 L_{\odot}$, respectively, while the radii are $R_{1}=3.5 \pm 0.3 R_{\odot}$ and $R_{2}=2.4 \pm 0.2 R_{\odot}$, respectively. These basic parameters are listed in Table 10 .

Our temperatures and luminosities of the 18 Vul components are compared with the solar-abundance evolutionary tracks of Girardi et al. (2000) in Figure 13. Both stars are on the main sequence. Masses estimated from the evolutionary tracks are also given in Table 10 .

\section{CIRCULARIZATION AND SYNCHRONIZATION}

Zahn (1977) and Tassoul \& Tassoul (1992) have produced very different theories for the way that binary orbits become circularized and binary components obtain rotational synchronization. Although their theories predict very different absolute timescales, they both lead to the conclusion that rotational synchronization occurs before orbital circularization. In an observational survey, Matthews \& Mathieu (1992) looked at 62 spectroscopic binaries with A-type primaries and periods less than 100 days. Circular or nearly circular orbits occurred for all the systems that have orbital periods $\lesssim 3$ days. However, even binaries with somewhat longer periods from 3 to 10 days often have circular orbits. HR 1528, 2 Sge, and 18 Vul with periods of 7.1,7.4, and 9.3 days, respectively, have circular or nearly circular orbits. HR 6993 with its longer 14.7 day period has a moderate eccentricity of 0.25 . Thus, the eccentricities of the four systems are consistent with the survey results of Matthews \& Mathieu (1992).

In an eccentric orbit, Hut (1981) has shown that the rotational angular velocity of a star will tend to synchronize with that of the orbital motion at periastron, a condition called pseudosynchronous rotation. With Equation (42) of Hut (1981) we compute a pseudosynchronous rotation period of 10.66 days for HR 6993.

To help in assessing component synchronization or pseudosynchronization in our four short-period binaries, we have determined projected rotational velocities of the components from our KPNO red wavelength spectra with the procedure of Fekel (1997). For A-type stars, the measured line broadening was converted to a $v \sin i$ value. For stars with later spectral classes, macroturbulent broadening has been taken into account. Following Fekel (1997) for late-F and G stars, a value of $3 \mathrm{~km} \mathrm{~s}^{-1}$ was used.

To convert the $v \sin i$ values into equatorial rotational velocities, we assume, as is generally done, that the axes of the orbital and rotational planes are parallel. If that is the case, then the two inclinations are equal, and we can adopt the orbital inclination as the rotational inclination.

\subsection{HR 1528}

To determine whether the components of HR 1528 are rotating synchronously, we first compute equatorial velocities from our projected rotational velocities. We then compare them with the predicted synchronous velocities. For the primary of HR 1528 , our $v$ sin $i$ value is $10.3 \pm 1.0 \mathrm{~km} \mathrm{~s}^{-1}$. Because of the secondary's weak lines, its value of $7.5 \pm 2.0 \mathrm{~km} \mathrm{~s}^{-1}$ has a greater uncertainty than that of the primary. From our comparison with the solar-abundance evolutionary tracks we adopt a mass of $1.95 M_{\odot}$ for the primary (Figure 13). That mass combined with its $m \sin ^{3} i$ value (Table 3 ) results in an orbital inclination of $66^{\circ}$. Adopting that inclination produces rotational velocities of 11.3 and $8.2 \mathrm{~km} \mathrm{~s}^{-1}$ for the primary and secondary, respectively. With our computed radii from the Stefan-Boltzmann law the synchronous rotational velocities are 19.4 and $12.2 \mathrm{~km} \mathrm{~s}^{-1}$ for the primary and secondary. The Am star primary is certainly rotating much more slowly than its synchronous value, while on the face of it the late-F or early$\mathrm{G}$ secondary is as well, although the large relative uncertainty, estimated for both its $v \sin i$ and especially its radius, makes synchronous rotation a possibility. For example, a radius of $1.2 R_{\odot}$, appropriate for a late-F dwarf, results in a predicted synchronous rotational velocity of $8.6 \mathrm{~km} \mathrm{~s}^{-1}$, a value in good agreement with our observed result. In Table 11 we have summarized the observed and predicted rotational velocities.

\subsection{HR 6993}

To determine if the components of HR 6993 are pseudosynchronously rotating, we initially compute their projected rotational velocities. From our KPNO spectra we determined $v \sin i$ values of $5.6 \pm 1.0$ and $5.9 \pm 1.0 \mathrm{~km} \mathrm{~s}^{-1}$ for the primary and secondary, respectively. With our previously computed pseudosynchronous rotation period of 10.66 days and the radii from the 
Table 11

Observed and Predicted Synchronous Rotational Velocities

\begin{tabular}{lccrc}
\hline \hline Parameter & HR 1528 & HR 6993 & 2 Sge & 18 Vul \\
\hline$v_{1} \sin i\left(\mathrm{~km} \mathrm{~s}^{-1}\right)$ & $10.3 \pm 1.0$ & $5.6 \pm 1.0$ & $9.4 \pm 1.0$ & $42.0 \pm 2.0$ \\
$v_{2} \sin i\left(\mathrm{~km} \mathrm{~s}^{-1}\right)$ & $7.5 \pm 2.0$ & $5.9 \pm 1.0$ & $9.8 \pm 1.0$ & $51.0 \pm 3.0$ \\
$v_{1}\left(\mathrm{~km} \mathrm{~s}^{-1}\right)$ & $11.3\left(i=66^{\circ}\right)$ & $11.2\left(i=30^{\circ}\right)$ & $12.9\left(i=47^{\circ}\right)$ & $44.2\left(i=72^{\circ}\right)$ \\
$v_{2}\left(\mathrm{~km} \mathrm{~s}^{-1}\right)$ & $8.2\left(i=66^{\circ}\right)$ & $11.8\left(i=30^{\circ}\right)$ & $13.4\left(i=47^{\circ}\right)$ & $53.6\left(i=72^{\circ}\right)$ \\
$v_{1}(\mathrm{sync})\left(\mathrm{km} \mathrm{s}^{-1}\right)$ & 19.4 & $16.1^{\mathrm{a}}$ & 13.7 & 19.0 \\
$v_{2}(\mathrm{sync})\left(\mathrm{km} \mathrm{s}^{-1}\right)$ & 12.2 & $13.3^{\mathrm{a}}$ & 11.0 & 13.0 \\
\hline
\end{tabular}

Note. ${ }^{\text {a }}$ Predicted pseudosynchronous rotational velocity.

Stefan-Boltzmann law, the pseudosynchronous rotational velocities for the primary and secondary are 16.1 and $13.3 \mathrm{~km} \mathrm{~s}^{-1}$, respectively. The minimum masses from our orbital solution are about $0.3 M_{\odot}$, the smallest values of our four systems. If we adopt a mass of $2.5 M_{\odot}$ for the primary from the comparison with solar-abundance evolutionary tracks in Figure 13, we obtain an orbital inclination of $30^{\circ}$. Assuming that the orbital and rotational inclinations are equal, then our $v \sin i$ values are doubled. This results in equatorial rotational velocities of 11.2 and $11.8 \mathrm{~km} \mathrm{~s}^{-1}$ for the primary and secondary, respectively. The value of the primary is still significantly less than its computed pseudosynchronous rotational velocity. While the value for the secondary is also less than its pseudosynchronous velocity, the uncertainties of the various quantities make it possible that it is rotating pseudosynchronously. The observed and predicted rotational velocities are compared in Table 11.

\subsection{Sge}

The orbit for the short-period pair of 2 Sge has an eccentricity of less than 0.01 , and so we simply examine the case for synchronous rotation of the components. Their projected rotational velocities from our KPNO spectra are $9.4 \pm 1.0$ and $9.8 \pm$ $1.0 \mathrm{~km} \mathrm{~s}^{-1}$ for the primary and secondary, respectively. With radii from the Stefan-Boltzmann law we determine synchronous equatorial rotational velocities of 13.7 and $11.0 \mathrm{~km} \mathrm{~s}^{-1}$. Again, comparing the minimum mass of the primary with its mass of $2.08 M_{\odot}$ estimated from evolutionary tracks (Figure 13), we obtain an inclination of $47^{\circ}$. Adopting this value increases the $v \sin i$ results to rotational velocities of 12.9 and $13.4 \mathrm{~km} \mathrm{~s}^{-1}$ for the primary and secondary, respectively. Thus, the primary of the short-period binary appears to be rotating synchronously. The secondary may be rotating faster than synchronous, although the predicted synchronous value and the observed value are uncertain enough that the secondary also may be synchronously rotating. We list the observed and predicted rotational velocities in Table 11.

For completeness sake, we note that the $v \sin i$ value of the close visual companion is $4 \mathrm{~km} \mathrm{~s}^{-1}$. Such a low value is consistent with that component being a late-F or early-G dwarf. Such stars are usually expected to be rotating slowly because of angular momentum loss.

\section{4. $18 \mathrm{Vul}$}

The $18 \mathrm{Vul}$ system also has a slight eccentricity of 0.01 , but again this value is so small that there is little difference between the synchronous and pseudosynchronous periods, and so we simply adopt the orbital period and discuss the possibility of synchronous rotation for the components of this system. The projected rotational velocities of both stars are at least four to five times greater than those of the other stars in this work, as we obtain $42 \pm 2$ and $51 \pm 3 \mathrm{~km} \mathrm{~s}^{-1}$ for the primary and secondary, respectively. With the radii from the Stefan-Boltzmann law, the synchronous velocities for the two components are 19.0 and $13.0 \mathrm{~km} \mathrm{~s}^{-1}$. In Figure 13, the position of the primary in the H-R diagram indicates a mass of $2.4 M_{\odot}$. That value, combined with its $m \sin ^{3} i$, results in an orbital inclination of $72^{\circ}$. Adopting that inclination, the equatorial rotational velocities become 44.2 and $53.6 \mathrm{~km} \mathrm{~s}^{-1}$ for the primary and secondary, respectively. Both values are substantially larger than the computed synchronous ones, so neither component is close to synchronous rotation. The observed and predicted rotational velocities are compared in Table 11.

\section{CONCLUSIONS}

We have determined improved orbital elements for four double-lined spectroscopic binaries, HR 1528, HR 6993, 2 Sge, and $18 \mathrm{Vul}$. The three systems with periods less than 10 days have circular or nearly circular orbits. Only HR 6933 with a period of 14.68 days has a moderate eccentricity. The very faint, close visual component of the 2 Sge short-period binary, which was discovered by the Hipparcos team (Perryman et al. 1997), is detected spectroscopically at red wavelengths. The minimum masses of the eight short-period binary components have been determined to an accuracy of $0.5 \%$ or better. Four of the eight components may be rotating synchronously or pseudosynchronously.

From the reanalyzed Hipparcos parallaxes (van Leeuwen 2007) and adopted masses of the systems, we estimate either the maximum angular separations of the components or in the case of the eccentric system HR 6993, the maximum angular nodal separation of the components (e.g., McAlister 1976; Halbwachs 1981). Similar to the results of Halbwachs (1981), we find that the separations all are in the 1-2 mas range, and therefore, although quite small, are still within the scope of modern optical interferometers. Thus, when our spectroscopic results are complemented with high-quality interferometric results, accurate three-dimensional orbits, masses, and distances for the systems will follow, allowing improved comparisons with evolutionary tracks.

The help of Daryl Willmarth in support of the KPNO coudé feed observations is appreciated. The research at Tennessee State University was supported in part by NASA, NSF, Tennessee State University, and the state of Tennessee through its Centers of Excellence program.

\section{REFERENCES}

Abt, H. A., \& Bidelman, W. P. 1969, ApJ, 158, 1091

Abt, H. A., \& Morrell, N. I. 1995, ApJS, 99, 135

Abt, H. A., \& Moyd, K. I. 1973, ApJ, 182, 809

Adams, W. S. 1912, ApJ, 35, 163

Adams, W. S. 1915, ApJ, 42, 172 
Appenzeller, I. 1967, PASP, 79, 102

Barden, S. C. 1985, ApJ, 295, 162

Barker, E. S., Evans, D. S., \& Laing, J. D. 1967, RGOB, 130, 355

Batten, A. H., Fletcher, J. M., \& MacCarthy, D. G. 1989, PDAO, 17, 1

Belmonte, J. A., \& Roca Cortés, T. 1989, IBVS, 3344, 1

Bertaud, C. 1970, A\&AS, 1, 7

Bertaud, C., \& Floquet, M. 1967, JO, 50, 425

Boden, A. F., Torres, G., \& Latham, D. W. 2006, ApJ, 644, 1193

Breger, M. 1969, ApJS, 19, 79

Cowley, A. P., Cowley, C. R., Jaschek, M., \& Jaschek, C. 1969, AJ, 74, 375

Crawford, D. L. 1979, AJ, 84, 1858

Cucchiaro, A., Macau-Hercot, D., Jaschek, M., \& Jaschek, C. 1978, A\&AS, 33, 15

Cunha, M. S. 2007, A\&ARv, 14, 217

Eaton, J. A., \& Williamson, M. H. 2004, Proc. SPIE, 5496, 710

Eaton, J. A., \& Williamson, M. H. 2007, PASP, 119, 886

Fabricius, C., \& Makarov, V. V. 2000, A\&A, 356, 141

Fekel, F. C. 1997, PASP, 109, 514

Fekel, F. C. 1999, in ASP Conf. Ser. 185, IAU Colloquium 170, Precise Stellar Radial Velocities, ed. J. B. Hearnshaw \& C. D. Scarfe (San Francisco, CA: ASP), 378

Fekel, F. C., Boden, A. F., Tomkin, J., \& Torres, G. 2009a, ApJ, 695, 1527

Fekel, F. C., Rajabi, S., Muterspaugh, M. W., \& Williamson, M. H. 2013, AJ, 145,111

Fekel, F. C., Tomkin, J., \& Williamson, M. H. 2009b, AJ, 137, 3900

Fekel, F. C., Tomkin, J., \& Williamson, M. H. 2010, AJ, 139, 1579

Fekel, F. C., Tomkin, J., Williamson, M. H., \& Pourbaix, D. 2011a, AJ, 142, 69

Fekel, F. C., \& Williamson, M. H. 2010, AJ, 140, 1381

Fekel, F. C., Williamson, M. H., \& Henry, G. W. 2011b, AJ, 141, 145

Floquet, M. 1975, A\&AS, 21, 25

Flower, P. J. 1996, ApJ, 469, 355

Geary, J. C., \& Abt, H. A. 1970, AJ, 75, 718

Girardi, L., Bressan, A., Bertelli, G., \& Chiosi, C. 2000, A\&AS, 141, 371

Gray, D. F. 1992, The Observation and Analysis of Stellar Photospheres (Cambridge: Cambridge Univ. Press)

Halbwachs, J. L. 1981, A\&AS, 44, 47

Harper, W. E. 1918, JRASC, 12, 392

Harper, W. E. 1932a, PDAO, 6, 79

Harper, W. E. 1932b, PDAO, 7, 1

Harper, W. E. 1935, PDAO, 6, 207

Huenemoerder, D. P., \& Barden, S. C. 1984, BAAS, 16, 510

Hummel, C. A., Carquillat, J.-M., Ginestet, N., et al. 2001, AJ, 121, 1623
Hut, P. 1981, A\&A, 99, 126

Johnson, H. L. 1966, ARA\&A, 4, 193

Lacy, C. H. S., \& Fekel, F. C. 2011, AJ, 142, 185

Levato, H. 1975, A\&AS, 19, 91

Levato, H., \& Abt, H. A. 1978, PASP, 90, 429

Lucy, L. B., \& Sweeney, M. A. 1971, AJ, 76, 544

Luyten, W. J. 1936, ApJ, 84, 85

Mason, B. D., Martin, C., Hartkopf, W. I., et al. 1999, AJ, 117, 1890

Matthews, L. D., \& Mathieu, R. D. 1992, in ASP Conf. Ser. 32, IAU Colloquium 135, Complimentary Approaches to Double and Multiple Star Research, ed.

H. A. McAlister \& W. I. Hartkopf (San Francisco, CA: ASP), 244

McAlister, H. A. 1976, PASP, 88, 317

McAlister, H. A., Hartkopf, W. I., Hutter, D. J., \& Franz, O. G. 1987, AJ, 93, 688

McCarthy, J. A., Sandiford, B. A., Boyd, D., \& Booth, J. 1993, PASP, 105,881

Osawa, K. 1959, ApJ, 130, 159

Perryman, M. A. C., Lindegren, L., Kovalevsky, J., et al. 1997, A\&A, 323, L49

Petrie, R. M. 1948, PDAO, 7, 415

Petrie, R. M. 1950, PDAO, 8, 319

Pintado, O. I., \& Adelman, S. J. 2003, A\&A, 406, 987

Pourbaix, D., Tokovinin, A. A., Batten, A. H., et al. 2004, A\&A, 424, 727

Quirrenbach, A. 2001, ARA\&A, 39, 353

Roberts, L. C. 2011, MNRAS, 413, 1200

Rodríguez, E., López de Coca, P., Rolland, A., Garrido, R., \& Costa, V. 1994, A\&AS, 106, 21

Scarfe, C. D., Batten, A. H., \& Fletcher, J. M. 1990, PDAO, 18, 21

Stickland, D. J. 1973, MNRAS, 161, 193

Stockton, R. A., \& Fekel, F. C. 1992, MNRAS, 256, 575

Stokes, N. R. 1972, MNRAS, 160, 155

Strassmeier, K. G., \& Fekel, F. C. 1990, A\&A, 230, 389

Svolopoulos, S. N. 1961, ApJ, 134, 612

Tassoul, J.-L., \& Tassoul, M. 1992, ApJ, 395, 259

Tomkin, J., \& Fekel, F. C. 2006, AJ, 131, 2652

Tomkin, J., \& Fekel, F. C. 2008, AJ, 135, 555

Torres, G., Andersen, J., \& Giménez, A. 2010, A\&ARv, 18, 67

van Leeuwen, F. 2007, Hipparcos, The New Reduction of the Raw Data (Dordrecht: Springer)

Wolfe, R. H., Horak, H. G., \& Storer, N. W. 1967, in Modern Astrophysics, ed. M. Hack (New York: Gordon \& Breach), 251

Young, R. K. 1917, JRASC, 11, 127

Zahn, J.-P. 1977, A\&A, 57, 383 